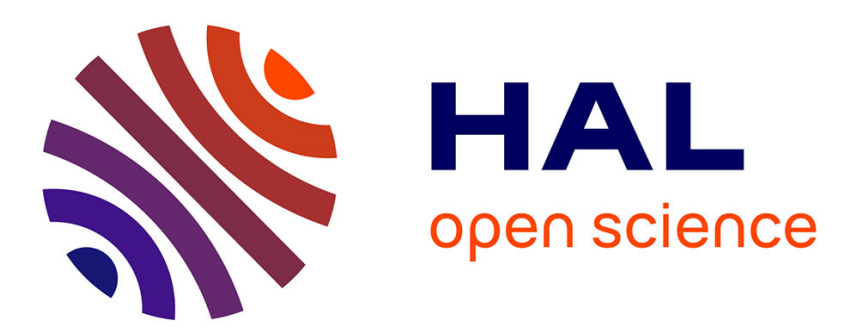

\title{
A study on amplitude transmission in ultrasonic welding of thermoplastic composites
}

\author{
Genevieve Palardy, Huajie Shi, Arthur Lévy, Steven Le Corre, Irene \\ Fernandez Villegas
}

\section{- To cite this version:}

Genevieve Palardy, Huajie Shi, Arthur Lévy, Steven Le Corre, Irene Fernandez Villegas. A study on amplitude transmission in ultrasonic welding of thermoplastic composites. Composites Part A: Applied Science and Manufacturing, 2018, 113, pp.339-349. 10.1016/j.compositesa.2018.07.033 . hal02372877

\section{HAL Id: hal-02372877 \\ https://hal.science/hal-02372877}

Submitted on 2 Dec 2019

HAL is a multi-disciplinary open access archive for the deposit and dissemination of scientific research documents, whether they are published or not. The documents may come from teaching and research institutions in France or abroad, or from public or private research centers.
L'archive ouverte pluridisciplinaire HAL, est destinée au dépôt et à la diffusion de documents scientifiques de niveau recherche, publiés ou non, émanant des établissements d'enseignement et de recherche français ou étrangers, des laboratoires publics ou privés. 


\title{
A STUDY ON AMPLITUDE TRANSMISSION IN ULTRASONIC WELDING
}

\section{OF THERMOPLASTIC COMPOSITES}

\author{
Genevieve Palardy ${ }^{3}$, Huajie Shi ${ }^{1}$, Arthur Levy², Steven LeCorre ${ }^{2}$ \\ and Irene Fernandez Villegas ${ }^{1}$ \\ ${ }^{1}$ Structural Integrity and Composites Group, Faculty of Aerospace Engineering, Delft University of \\ Technology, Kluyverweg 1, 2629 HS Delft, The Netherlands. \\ ${ }^{2}$ Laboratoire de Thermocinétique de Nantes, La Chantrerie, rue Christian Pauc, BP 90604, 44306 \\ Nantes Cedex 3, France. \\ ${ }^{3}$ Department of Mechanical \& Industrial Engineering, Louisiana State University, 3261 Patrick F. \\ Taylor Hall, Baton Rouge, LA 70803, United States.
}

\begin{abstract}
Ultrasonic welding of thermoplastic composite materials is a promising joining technique that is now moving towards up-scaling, i.e. the assembling of large industrial parts. Despite its growing technological maturation, the assumed physical mechanisms underlying ultrasonic heating (viscoelastic heating, friction) are still insufficiently understood and modelled. In particular, the hammering phenomenon, resulting from the periodic loss of contact between the sonotrode and adherends due to the high frequency vibration caused to the former, directly impacts the heating efficiency. We propose in this work an original experimental and modelling approach towards a better understanding of the hammering effect. This approach makes combined use of: (i) an experimental static welding setup provided with a high-frequency laser sensor to analyse the vibration amplitude transmitted to the adherends and (ii) an improvement of the multiphysical finite element model already presented in previous works. Results show it is possible to obtain a good estimation of the vibration transmitted to the upper adherend from laser measurements close to the sonotrode. The hammering effect is shown to decrease during the welding process, due to the heating of the interface which directly affects further
\end{abstract}


heat generation. Quantitative introduction of this hammering effect in the existing numerical model results in improved predictions in terms of dissipated power in time.

\section{Keywords:}

A/ Thermoplastic resin; B/ Vibration; C/ Process Modelling; E/ Joints/Joining.

\section{Introduction}

Composite materials, in particular carbon fibre reinforced polymers (CFRP), have been increasingly used for applications in aircraft structures due to their great potential for weight reduction [1]. As opposed to thermoset composites, and as a result of the linear and entangled molecular structure of thermoplastics, thermoplastic composites (TPCs) can be re-melted and re-shaped easily. It is possible to manufacture and join TPC components using low-cost manufacturing technologies, such as press forming or welding. Since only local heating of the structure is required, welding processes are usually faster, more energy efficient and less expensive, which makes thermoplastic composites attractive for use in the aerospace industry.

Ultrasonic welding (USW) is a very efficient technique for TPCs with fast welding times $(<5 \mathrm{~s})$, great potential for mass production and does not need introduction of foreign material at the weldline $[2,3]$. The principle of USW is shown in Figure 1. An energy director (ED) made of the same thermoplastic as the adherends' matrix is placed between the lower and upper adherends. In this work, instead of the classical triangular shapes, flat energy directors, which consist of thin films, are used [4]. A sonotrode applies pressure on the parts to be welded, as well as high frequency (20- 
$40 \mathrm{kHz}$ ) and low amplitude vibrations (2.5-250 $\mu \mathrm{m}$ peak-to-peak amplitude) [5]. Heat generation is localized at the interface due to the lower compressive stiffness of the energy director. The welding energy is generated from a combination of surface friction and viscoelastic heating [6]. A consolidation phase follows the vibration phase to create a high quality bond.

There is considerable research literature showing experimental investigations of the USW process for TPCs at the coupon level $[4,5,7-15]$. These studies involve understanding the effect of various parameters such as welding force, vibration amplitude, solidification force and time and shape of the energy director on the welding process and on the quality of the welded joints. While experimental research provides solid insight into the inner workings of ultrasonic welding of thermoplastic composites, the complex heating mechanisms behind the process remain insufficiently understood and hence not accurately modelled. As USW is moving toward up-scaling and industrialization, the development of a reliable finite element model has become essential in increasing the robustness and reliability of sequential and even continuous welding of larger and more complex parts [16-18].

To date finite element analyses of the USW process for TPCs are mostly limited to prediction of the temperature field at the weld interface and in the adherends based on the shape of the energy director $[6,19-22]$. However, obtaining accurate measurements of temperature distribution at the welding interface and within the adherends is difficult and hence experimental validation of such models remains a challenge. More recently, Levy et al. developed a numerical multiphysical model to predict power dissipation during the initial transient heating phase of the USW 
process, which was also applied to flat energy directors $[23,24]$. This approach is particularly attractive since the power dissipated during the USW process is affected by the physical changes occurring at the welding interface [7] and is provided as a power-time curve by microprocessor-controlled ultrasonic welders. Consequently, dissipated power throughout the welding process constitutes a meaningful and relatively straightforward data source for experimental validation of USW numerical models. Coupling the physics of adhesion, heat transfer and solid mechanics showed promising results, but several empirical parameters had to be estimated to obtain good agreement with the experimental data [23]. One of those parameters was the hammering coefficient, $\alpha_{h}$. Hammering is the loss of contact between sonotrode and upper adherend during the vibration phase of the USW process [25]. As a result, it leads to reduced friction and viscoelastic dissipation and is therefore critical for heat generation at the weld interface.

As previously mentioned, heat is generated through frictional and viscoelastic heating during USW. It is thought that frictional dissipation $(Q \quad)$ at the interface between the energy director and the adherends mostly occurs before the glass transition temperature is reached. It can be calculated from the average dissipated mechanical work associated with friction during an ultrasonic cycle, given by Eq. (1) $[6,23]:$ 
where $e$ is called the hammering efficiency, i.e. the ratio between actual heat generation (with hammering) and ideal heat generation (without hammering), is the pulsation of vibration, is the vertical stress on the horizontal interface and is the horizontal displacement discontinuity across the interface. Viscoelastic heating, which is maximized around the glass transition temperature of the thermoplastic polymer, can be quantified, based on the work by Tolunay et al. ([26]), with Eq. (2):

where is the loss modulus of the material and $\varepsilon$ is the amplitude strain tensor. In previous work [23], the hammering efficiency, e, i.e. the ratio between the actual heat generation (with hammering) and the ideal heat generation (without hammering), was incorrectly written as, where was defined as the hammering coefficient. The hammering coefficient accounted for the amplitude transmitted between the sonotrode and the upper adherend according to the following equation, where is the amplitude transmitted to the substrate and is the amplitude of the sonotrode:

It should be noted that for the sake of clarity is renamed in the present paper as amplitude transfer ratio. In appendix A of the present paper, it is shown that in fact, . Despite its importance on frictional and viscoelastic heat generation during the USW welding process, neither nor $e$ have ever been experimentally measured. 
While the approach proposed by Levy et al. [23] is an important first step toward successful implementation of the physics behind USW of TPCs, it can benefit from empirical evaluation and quantification of the hammering effect. To further improve the proposed model and achieve closer agreement with the experimental data, it is necessary to properly determine the actual amplitude transmitted to the parts being welded, as it can greatly influence heat generation and hence power dissipated during the process. Therefore, in this study, we proposed and assessed a strategy to measure and estimate amplitude transmission to the upper adherend during ultrasonic welding of thermoplastic coupons focusing on the vibration phase of the USW process. The experimental measurements were used as input into a COMSOL Multiphysics model to simulate dissipated power during the vibration phase of USW for a specific welding force and estimated transmitted amplitude. The predicted dissipated power was then compared to the experimental power curve provided by the ultrasonic welder.

\section{Methods}

In this paper, a combination of experimental and finite element analysis approaches were employed. The experiments consisted of composite adherends manufacturing and welding, as well as laser sensor measurements of amplitude transmission on the surface of the upper adherend. A numerical multiphysical model, based on the framework developed by Levy et al. [23], was used to predict the power curves obtained from the ultrasonic welding process by taking into consideration the amplitude transmission measurements. 


\subsection{Experimental}

Laminates were manufactured from six layers of carbon fibre/polyetherimide (CF/PEI) 5 harness satin T300 prepreg, provided by TenCate, The Netherlands. They were stacked with a $[0 / 90]_{3 S}$ sequence and consolidated in a hot-platen press at $320^{\circ} \mathrm{C}$ and $2 \mathrm{MPa}$ for $30 \mathrm{~min}$. After consolidation, the laminates were water-jet cut into $101.6 \mathrm{~mm}$ by $25.4 \mathrm{~mm}$ adherends for the welding process. A rectangular neat PEI film, with a thickness of $0.25 \mathrm{~mm}$, was used as the energy director. Before welding, the composite adherends and energy directors were dried in an oven at $135^{\circ} \mathrm{C}$ for 6 hours and 1 hour, respectively.

A $20 \mathrm{kHz}$ Rinco Dynamic microprocessor controlled ultrasonic welder with a maximum power output of $3000 \mathrm{~W}$ was used to weld specimens in a single lap shear configuration with an overlap area of $12.7 \mathrm{~mm}$ x $25.4 \mathrm{~mm}$. A rectangular sonotrode with a surface area of $14.9 \mathrm{~mm} \times 30.1 \mathrm{~mm}$ and a gain (i.e. amplification of the ultrasonic wave in the sonotrode) of 2.75 was used. A custom-made welding jig was used to clamp the lower and upper adherends during welding (Figure 2). It prevented in-plane movement but allowed the upper adherend to remain perfectly parallel to the bottom one during the whole process. A welding force of $1500 \mathrm{~N}$ and a vibration amplitude amounting to $36.3 \mu \mathrm{m}$ (half peak-to-peak value), were used for measurements during the welding process. The duration of the vibration phase of the USW process was indirectly controlled by the displacement of the sonotrode, called "travel". The optimum travel was selected as $60 \%$ of the energy director thickness (i.e. $0.15 \mathrm{~mm}$ for a $0.25 \mathrm{~mm}$-thick energy director), following the empirical procedure detailed in [7]. After welding, the joints underwent a solidification phase with a 
consolidation force of $1000 \mathrm{~N}$ applied for 4 seconds. It should be noted that the parameters of the welding process as well as the thickness of the adherends, corresponding to typical values for ultrasonic welding of thermoplastic composites, were not modified in this study, since its main focus was the amplitude transmission during welding. Detailed results on the effect of the welding parameters and the thickness of the adherends on the welding process and its outcome can be found in [7]. It should also be noted that even though the nominal vibration frequency of the ultrasonic welder used in this study was $20 \mathrm{kHz}$, slight variations (within approximately a 1\% range) in the actual frequency (provided by the welder after the welding process) are typically observed from weld to weld. The exact impact of those frequency variations in the welding process is unknown although it is assumed to be small owing to the consistent welding output.

As previously described in Section 1, when hammering occurs during the application of ultrasonic vibration, there is a loss of contact between the sonotrode and the upper adherend. To determine the actual amplitude transmitted from the sonotrode to the weld assembly, the displacement of the upper adherend was measured with a laser displacement sensor. A CMOS sensor by KEYENCE®, LK-H052 sensor (range $\pm 10 \mathrm{~mm}$, accuracy $0.025 \mu \mathrm{m}$ ) with LK-G5000P controller, was used in this study. The laser sensor was set to work at a sampling rate of $200 \mathrm{kHz}$ for a maximum recording time of 6 seconds. More specifically, 10 points could be measured in each vibration cycle since the welder worked at a frequency of $20 \mathrm{kHz}$. The accuracy of the sensor was assessed by measuring the displacement at the base the sonotrode for a certain nominal vibration amplitude. For these tests, referred to 
hereafter as sensor calibration tests, the sonotrode was vibrated freely in the air and the laser sensor was placed perpendicular to the base of the sonotrode.

For maximum accuracy in the measurement of the vibration transmitted to the upper adherend during the ultrasonic welding process, displacement measurements on the adherend should be taken directly underneath the sonotrode, but since this was physically not possible, displacement measurements were taken at different distances $d$ from the sonotrode to evaluate the impact of $d$ on the sensor measurements and find the most appropriate $d$ value (Figure 3). As depicted in the welding schematic and displacement measuring setup used in this work and shown in Figure 3, the laser beam had to be placed at an angle $\alpha$ from the overlap in order to allow small $d$ values. Since in this setup the vertical vibrational movement of the composite adherend was not parallel to the direction of the laser beam, the measured displacement $D_{\text {meas }}$ was converted to the real displacement $D$ by using Eq. (4):

$$
D=D_{\text {meas }} * \cos (\alpha)
$$

By comparing the measurement results for a constant movement from a vertically installed sensor and a tilted sensor at $0^{\circ} \leq \alpha \leq 60^{\circ}$, it was found that the error introduced when $\alpha \leq 45^{\circ}$ was less than $1.6 \%$ and increased when $\alpha>45^{\circ}$. Therefore, in order to measure the displacement as close as possible to the overlap, a $45^{\circ}$ was selected as the sensor tilt angle. 


\subsection{Modelling}

In this paper, a 2D COMSOL Multiphysics $5.2 ®$ heat transfer model, previously developed in [23], was improved and subsequently used to predict the welding power dissipation, i.e. power curves, during the USW process. Figure 4 shows that only a quarter of the actual geometry was considered in the model (one adherend and half the thickness of the energy director) in order to reduce computing time. It should be noted that such symmetric system is a simplification and does not accurately reflect the geometry of the welding set-up and specimen. However it is based on the assumption that the welding overlap (which does have two axes of symmetry) is the main source of power dissipation during the welding process. The assumption also implies that the friction behavior of the two adherend/energy director interfaces is the same, thus disregarding any potential instability of the friction behavior. The symmetry assumption could hence be a source of errors in the numerical prediction and should be addressed in future work. The present study focused on the heating of both the energy director and the adherend, but did not account for the flow that occurs at the interface upon melting of the energy director and adherend. The model takes into consideration viscoelastic dissipation ((2), as well as friction, intimate contact evolution and adhesion evolution at the interface between the energy director and the adherend.

In order to improve on the previous modelling framework presented in [23], three main changes were applied: 1) the amplitude transmission from the sonotrode to the upper adherend was experimentally determined (in this paper) and was used instead of the previously used hammering coefficient, 2) viscoelastic heating from both the 
energy director and the adherends was taken into account, and 3) the simulation was stopped when the minimum temperature in the ED exceeded $T_{g}+100^{\circ} \mathrm{C}\left(315^{\circ} \mathrm{C}\right)$ in order to capture the effect of viscoelastic heating over a representative duration of the vibration phase of the welding process $(\sim 0.7 \mathrm{~s},[7])$. The model parameters and material properties are detailed in [23]. 


\section{Results and discussion}

\subsection{Sensor calibration measurements}

As mentioned before, calibration of the laser displacement sensor was performed by applying a known vibration amplitude hereafter referred to as nominal amplitude, to the sonotrode and comparing it to the vertical displacement measured on the bottom surface of the sonotrode by the laser sensor. In these calibration tests, the sonotrode was vibrating freely in the air and the laser sensor was placed perpendicular to the bottom surface of the sonotrode. It should be noted that no additional information about the actual vibration amplitude was available in this study, hence the measurements of the laser sensor could only be compared to the nominal amplitude entered in the ultrasonic welder. Sensor displacement measurements were taken at five different positions (three measurements per position), as illustrated in Figure 5. Sonotrode displacement measurements at position 1 for a nominal amplitude of $45.4 \mu \mathrm{m}$ and a duration of $0.5 \mathrm{~ms}$ are shown in Figure 6(a) as a representative example of the results of the calibrations tests. The measured average amplitude over all positions ( 1 to 5 ) was $44.9 \pm 2.3 \mu \mathrm{m}$, thus displaying close agreement (within 1.2\%) with the nominal value, $45.4 \mu \mathrm{m}$. Furthermore, Figure 6(b), corresponding to measurements at a different position, demonstrates that the measured oscillation frequency obtained through Fast Fourier Transform (FFT) was similar to the nominal value given by the supplier of the ultrasonic welder, $20 \mathrm{kHz}$. Note that the differences in amplitude between Figure 6(a) and Figure 6(b) correspond to the variability observed in measurements taken at different positions in the bottom surface of the sonotrode $(44.9 \pm 2.3 \mu \mathrm{m})$. These results proved the suitability 
of the chosen laser sensor to provide accurate measurements of the ultrasonic vibrations during the USW process despite the relatively low number of points (10 points) captured during each vibration cycle. It should be noted that owing to the fact that the calibration tests were conducted independently from the research on amplitude transmission during welding, the nominal amplitude value used for the calibration tests $(45.4 \mu \mathrm{m})$ was not the same as the nominal amplitude used in the welding tests $(36.3 \mu \mathrm{m})$. Nevertheless based on the results of the calibration tests and taking into account the accuracy of the laser sensor $(0.025 \mu \mathrm{m})$, the latter was assumed to be a suitable system to measure transmitted amplitude during welding.

\subsection{Displacement measurements for upper adherend}

As explained in Section 2.1, the amplitude transmission between the sonotrode and the upper adherend was estimated through measurement of the vertical displacement of the latter during the USW process. As shown in Figure 7, such measurements were carried out at different distances $d$ from the sonotrode to identify the position leading to the highest accuracy. The $d$ values were $2 \mathrm{~mm}, 5 \mathrm{~mm}, 15 \mathrm{~mm}$ and $25 \mathrm{~mm}$. Measurements were repeated three times for each distance $d$.

Representative displacement curves are presented in Figure 8 for (a) 2 mm, (b) 5 $\mathrm{mm}$, (c) $15 \mathrm{~mm}$, and (d) $25 \mathrm{~mm}$. The blue curves, corresponding to the data obtained from the laser sensor measurements, were compared to the displacement curves provided by the ultrasonic welder (i.e. vertical displacement of the sonotrode relative to its initial position at the onset of the vibration phase) for each case (dashed, red line). Generally, there was good agreement in the trend shown by both curves consisting of an initial plateau (corresponding to the heating of the energy director 
[7]) followed by a steady downward displacement of the sonotrode (corresponding to the squeeze flow of the energy director under the welding pressure [7]). It should be noted that whereas the displacement measured by the laser sensor was able to capture the high-frequency oscillation, the displacement measured by the welder did not capture this oscillation. The average amplitude over the entire duration of the vibration phase for different $d$ values was calculated and compared to the nominal amplitude of the welding machine $(45.4 \mu \mathrm{m})$ as well as to numerical calculations, as shown in Figure 9. The numerical calculations were obtained from simplified dynamic numerical simulations, performed with COMSOL Multiphysics $5.2 \AA$, for the boundary value problem described in Figure 4. In these simulations, the mechanical properties of the adherends and energy director were taken from [23] (values at room temperature). To simulate the hammering effect in a simplified manner, an intermittent sinusoidal force at $\omega=20 \mathrm{kHz}, F(t)$, was applied on the upper adherend just under the sonotrode, as described by Eq. (5):

This analytical form only considers the negative part of the applied force, i.e. force applied on the adherend, which combines a constant contribution (holding force $F_{0}$ amounting to $1500 \mathrm{~N}$ ) and a fast oscillating contribution, $F_{1} \sin (\omega t)$. The parameter $F_{1}$, equal to $66 \mathrm{kN}$, was estimated from the normal stress necessary to impose the nominal amplitude of the vibration onto the welding stack, i.e. adherends and energy director [23]. It should be noted that owing to the relatively small thickness of the welding stack the oscillating contribution to the force was significantly higher than the static contribution as discussed in [23]. 
Several observations can be made from the data plotted in Figure 9. First and foremost, the distance from the sonotrode had a strong effect on the amplitude transmission measurements. The experimental and the numerical amplitude values showed a similar trend characterized by a non-uniform distribution of the vibration amplitude along the upper adherend. This indicates that, for the specific set of boundary conditions considered in this study, the superposition of the different vibration modes excited by the sonotrode in the upper adherend played a major role on the effect of the distance from the sonotrode on the measured amplitude. Deformation of the springs beneath the sliding platform onto which the upper adherend was clamped (not considered in the numerical calculations) was also believed to contribute to the observed effect. Secondly, the numerical results indicated that the average amplitude at $d=2 \mathrm{~mm}$ could be considered relatively close to the average amplitude directly underneath the sonotrode. Consequently, $d=2 \mathrm{~mm}$ was selected as the most appropriate location for the transmitted amplitude measurements.

The average amplitude measured at $d=2 \mathrm{~mm}$ was $42.0 \pm 8 \mu \mathrm{m}$ which was also relatively close to the nominal amplitude of the sonotrode, $45.4 \mu \mathrm{m}$. The scatter in these measurements was high, with a coefficient of variation amounting to almost $20 \%$, but still lower than the overall scatter in measurements obtained at $d>2 \mathrm{~mm}$. Since, as shown in Figure 9, the amplitude measured at $d=2 \mathrm{~mm}$ could be expected to be close to but not the same as right at the welding overlap, simulation could eventually enable to define a quantitative correction factor to estimate the transmitted amplitude at the welding overlap, i.e. right underneath the sonotrode, 
from the measurements taken at $\mathrm{d}=2 \mathrm{~mm}$. This would however require fine tuning of all simulation parameters and is beyond the scope of this paper. Moreover the FFT of the displacement measurements at $d=2 \mathrm{~mm}$ (see Figure 10) showed, in addition to a main peak at $20 \mathrm{kHz}$, secondary peaks attributed to the different vibration models in the upper adherend, at approximately 40, 60 and $80 \mathrm{kHz}$. These secondary frequencies were, however, not considered when using the displacement measurements at $\mathrm{d}=2 \mathrm{~mm}$ to estimate the transmitted amplitude at the welding overlap.

\subsection{Strategy to determine amplitude transmission}

As briefly explained in Section 1, the coefficient of hammering, $\alpha_{h}$, can be expressed as a function of the duration of the actual contact between the sonotrode and the upper adherend. However, experimentally determining this duration of contact from laser sensor data alone can be particularly complex. Therefore, the alternative and more straightforward approach followed in this paper entailed considering the hammering coefficient as the ratio between the actual amplitude transmitted to the upper adherend, i.e. the compressive displacement induced in the adherend by the sonotrode, and the nominal amplitude in the sonotrode.

To simplify the laser sensor data (shown in Figure 8) and facilitate its input into the 2D model described in Section 2.2, a strategy to estimate the amplitude transmitted to the upper adherend was developed. Figure 11 (a) shows representative laser sensor data $(d=2 \mathrm{~mm})$ for the vibration phase in a welding process with a force of $1500 \mathrm{~N}$ and a nominal amplitude of $36.3 \mu \mathrm{m}$. First, as indicated 
by the dashed line in the close-up in Figure 11 (b), a curve was fitted through the data points to establish the average sonotrode displacement. Then, the transmitted amplitude was calculated by taking half its peak-to-peak displacement value as shown by the arrow in Figure 11 (b). Due to the large amount of measured data points, the amplitude values were computed for time intervals of $0.004 \mathrm{~s}$, as shown in Figure 12. This corresponds to intervals of about 80 ultrasonic periods and ensures that the true peak is captured. To circumvent the high variability of the data points and to apply it to the numerical model, the data was fitted using a linear function (Figure 12). Considering that the ultrasonic welder used in this study, establishes full vibration in the sonotrode in approximately $0.05 \mathrm{~s}$, the piecewise affine function shown in Figure 12 was defined for the amplitude transmission. According to this function, the amplitude transmission increased sharply from 0 to $19.7 \mu \mathrm{m}$ within the first $0.05 \mathrm{~s}$, and then increased gradually until it reached $33.0 \mu \mathrm{m}$ at the end of the vibration phase of the welding process.

It is interesting to note that the amplitude transmission seemed to improve over time, meaning that hammering decreased. It is believed that after the initial establishment of the vibrations, the decrease in stiffness, caused by heating and melting of the energy director at the interface, improved the compliance between the sonotrode and upper adherend, hence promoting a better transmission of amplitude.

\subsection{Implementation into numerical multiphysical model}

In the 2D COMSOL model presented in Section 2.2, the hammering coefficient $\alpha_{h}$ was implemented as the ratio between the bilinear function shown in Figure 12 and 
the nominal amplitude of the sonotrode, i.e. $36.3 \mu \mathrm{m}$, over the duration of the vibration phase of the welding process $(0.7 \mathrm{~s})$. The reader should note that the hammering efficiency $e$ in equations (1) and (2) was taken as equal to as justified in appendix A. Figure 13 shows representative experimental and simulation results for the case shown in Section 3.3: $1500 \mathrm{~N}$ welding force and $36.3 \mu \mathrm{m}$ nominal amplitude. The "Exp power" curve was obtained from the welding machine. The simulation results included four different power curves: frictional heating between the energy director and adherends ("Friction heating"), viscoelastic heating from the energy director ("ED Viscoelastic heating"), viscoelastic heating from the adherends ("Ad Viscoelastic heating") and the sum of all heating sources resulting in the "Total heating" curve.

When comparing total simulated power to experimental power, it generally followed a similar behaviour: two main power peaks (circled areas for both simulated and experimental curves) with a maximum power value of between 1300 and $1600 \mathrm{~W}$. As shown experimentally, the first peak coincides with the moment at which melting first occurs within the energy director [8]. The second power peak was associated with melting occurring within the composite adherends [8]. Similarly, the first power peak in the simulated power curve was, as seen in Figure 13, mostly attributed to the viscoelastic heating in the energy director, with secondary contributions from viscoelastic heating of the adherends and frictional heating. Likewise, the second power peak in the simulated curve was clearly a result of the viscoelastic heating of the adherends. The delay observed between the occurrence of simulated and experimental peaks may have been caused by assuming in the model that energy 
dissipation was purely concentrated at the interface. In reality, part of the welding energy is dissipated in the welding setup, tooling and anvil, as well as in the adherends outside the overlap, which means it would take more time for the weld to be created.

It should be noted that the shape of the first peak in the simulated power curve is likely influenced by the loss modulus, $E^{\prime \prime}$, in $\left(2\right.$, which is dominant around $T_{g}$ $\left(\sim 215^{\circ} \mathrm{C}\right)[23]$. A closer examination of the predicted temperature profile across the thickness of the energy director and upper adherend at $0.12 \mathrm{~s}$ (Figure 14 (a)), i.e. the time at which a distinct and very sharp power peak occurs, revealed that at that moment, the temperature in the ED was between $200^{\circ} \mathrm{C}$ and $250^{\circ} \mathrm{C}$, which indicated the predominance of viscoelastic heating over frictional heating. Regarding the second peak in the simulated power curve, occurring at around $0.50 \mathrm{~s}$, the predicted temperature profile clearly showed that temperature values around $T_{g}$ were expected within the upper adherend, contributing to viscoelastic heat generation within the adherend itself (Figure 14 (b)).

Improvement over the previous version of the model presented in [23] can be highlighted in two ways. Firstly, the total simulated power values were at a similar level as the experimental ones without the use of a global efficiency factor and both the simulated and experimental curves showed two main power peaks. This was achieved by a combination of better empirical determination of the amplitude transmitted to the upper adherend (no hammering coefficient required) and consideration of the contribution of viscoelastic heating from the adherends. Secondly, the temperature evolution was generally captured accurately as both power 
peaks coincided with the moment when $T_{g}$ was first reached in the ED and secondly, in the adherend.

Nevertheless, the simulated results still show discrepancies with the experimental results, one of the most significant ones being the inability of the model to accurately predict the time at which the main power peaks occurred causing the simulated and experimental power curves to not overlap. Discrepancies between simulated and experimental results are believed to be a consequence of assumptions and limitations of the model. For instance, the model was limited by the fact that flow, which in the experiment shown in Figure 13 was observed to commence at around $0.5 \mathrm{~s}$, was not taken into consideration. Flow should be considered in future iterations of this model in order to fully capture heat generation and heat transfer in the ultrasonic welding process. Likewise, the low values of friction heating compared to viscoelastic heating, even at the onset of the welding process seem to contradict experimental findings [6], which suggests that a revision of the approach used in the friction heating model might be needed.

\section{Conclusions}

In this paper, an experimental determination of the amplitude transmitted to the upper adherend during ultrasonic welding of thermoplastic composites was carried out. The experimental results were used to improve upon a previously developed COMSOL Multiphysics model by providing more accurate input parameters regarding the hammering effect. Based on both experimental and modelling findings, the following observations can be made: 
- When using the laser sensor on the surface of the upper adherend, measurement data varied significantly when the distance between the sonotrode and the sensor increased. In order to obtain measurements similar to what the upper adherend experienced underneath the sonotrode, it was recommended to place the sensor as close as practically possible to the sonotrode (i.e. $2 \mathrm{~mm}$, in our case).

- The amplitude transmitted to the upper adherend was calculated and averaged at selected points over the duration of the process. It was described by a bilinear function, with positive slopes, indicating less hammering toward the end of the process cycle. This behaviour suggested that after onset of the vibrations, the decrease in stiffness of the energy director, induced by heating and melting at the interface, led to an improved compliance between the sonotrode and upper adherend, hence promoting better transmission of amplitude.

- The experimental results were implemented into a numerical multiphysical model as the imposed sonotrode displacement for prediction of dissipated power based on frictional and viscoelastic heating. Improved agreement between simulated power and experiments was achieved. Taking into consideration viscoelastic heating from both the energy director and the adherends revealed the presence of two power peaks corresponding to the main stages of the ultrasonic welding process in thermoplastic composites. To further improve this model, flow of the energy director at the interface should be taken into consideration, so that physical behaviour of the polymer past $T_{g}$ 
can be represented more realistically. Moreover the friction heating models should be revised. 


\section{Appendix A}

In previous works on modelling and simulation of the process by the same group $[19,20,23,24]$, the dissipated power (dissipated by viscoelasticity or by friction) used as a source term in the heat transfer problem, was obtained from the elastic problem. The load was considered sinusoidal, and the stresses and strains integrated over an ultrasonic period. In order to account for the hammering effect, an approximate correction coefficient was used in the expression of the source terms. In this appendix, this correction factor is theoretically derived from the amplitude effectively transmitted to the upper adherend.

The sonotrode tip has a displacement that is sinusoidal as shown in (A1):

where is the amplitude, the pulsation, and $f$ the frequency usually in the order of $20 \mathrm{kHz}$. Because of the hammering effect and the loss of contact between the sonotrode and the composite, the imposed displacement on the top surface of the top adherend is not equal to . Rather, it is a truncated sine as illustrated in Figure A1. The contact time ratio can be defined as:

where $t_{c}$ is the loss of contact time during an ultrasonic period $2 \pi \omega$. ranges between 0 and 1 and reaches 1 for a perfect contact with no hammering.

The amplitude of the imposed displacement fulfils: 
where $\alpha_{h}$ is the amplitude transfer ratio which is the ratio between the amplitude transmitted to the substrate $a_{a d h}$ and the sonotrode amplitude $a_{\text {sono. }}$.

For the sake of clarity, let us consider the average viscoelastic dissipation $Q_{v e}$ per period. When there is no contact, the dissipated power is zero. Thus:

$$
Q_{v e}=\frac{1}{T} \int_{t=t_{c} / 2}^{T-t_{c} / 2} \sigma: \dot{\varepsilon}
$$

where $\sigma$ is the Cauchy stress tensor and $\dot{\varepsilon}$ the strain rate tensor. Following the methodology developed in previous works [5],[26], under linear viscoelasticity assumptions, this becomes:

$$
Q_{v e}=\frac{2 E^{\prime \prime} \omega \varepsilon_{0}: \varepsilon_{0}}{T} \int_{t=t_{c} / 2}^{T / 2} \sin ^{2}(\omega t)
$$

where $\varepsilon_{0}$ is the amplitude of the strain tensor obtained when solving the elastic problem with the sonotrode displacement $u_{\text {sono }}$. Integrating the squared sinus and noting that $\omega=2 \pi / T$ leads to:

$$
Q_{v e}=\frac{2 E^{\prime \prime} \omega \varepsilon_{0}: \varepsilon_{0}}{T}\left(\frac{t_{c}}{T}-\frac{\sin \left(2 \pi t_{c} / T\right)}{2 \pi}\right)
$$

which corresponds, when using the contact time ratio $\alpha_{t}$, to:

$$
Q_{v e}=Q_{v e}^{*}\left(\alpha_{t}+\frac{\sin \left(2 \pi \alpha_{t}\right)}{2 \pi}\right)
$$

In this final equation, $Q_{v e}^{*}=2 E^{\prime \prime} \omega \varepsilon_{0}: \varepsilon_{0} / T$ denotes the reference viscoelastic dissipation obtained theoretically if no hammering occurs (i.e. the dissipation obtained in previous works). The viscoelastic efficiency $e$ of the process is thus:

$$
e=\alpha_{t}+\frac{\sin \left(2 \pi \alpha_{t}\right)}{2 \pi}
$$

which can be expressed as a function of the amplitude transfer ratio $\alpha_{h}$ using Eq. (A2). Figure A2 shows the dependency of the efficiency $e$ versus the amplitude transfer ratio $\alpha_{h}$. In first approximation, one may choose: 
which is different from what was given in previous works that assumed . Selfheating efficiency can therefore be read directly from amplitude measurements as proposed in this work. 


\section{References}

[1] A.K. Kaw, Mechanics of Composite Materials, 2nd ed., CRC Press2005.

[2] C. Ageorges, L. Ye, M. Hou, Advances in fusion bonding techniques for joining thermoplastic matrix composites: a review, Composites Part A: Applied Science and Manufacturing 32(6) (2001) 839-857.

[3] A. Yousefpour, M. Hojjati, J.-P. Immarigeon, Fusion Bonding/Welding of Thermoplastic Composites, Journal of Thermoplastic Composite Materials 17(4) (2004) 303-341.

[4] I.F. Villegas, B. Valle Grande, H.E.N. Bersee, R. Benedictus, A comparative evaluation between flat and traditional energy directors for ultrasonic welding of CF/PPS thermoplastic composites, Compos. Interfaces 22(8) (2015) 717-729.

[5] A. Benatar, T.G. Gutowski, Ultrasonic welding of PEEK graphite APC-2 composites, Polymer Engineering \& Science 29(23) (1989) 1705-1721.

[6] Z. Zhang, W. Xiaodong, L. Yi, Z. Zhenqiang, W. Liding, Study on Heating Process of Ultrasonic Welding for Thermoplastics, Journal of Thermoplastic Composite Materials 23(5) (2010) 647-664.

[7] I.F. Villegas, Strength development versus process data in ultrasonic welding of thermoplastic composites with flat energy directors and its application to the definition of optimum processing parameters, Composites Part A: Applied Science and Manufacturing 65 (2014) 27-37.

[8] I.F. Villegas, In situ monitoring of ultrasonic welding of thermoplastic composites through power and displacement data, Journal of Thermoplastic Composite Materials 28(1) (2015) 66-85.

[9] I.F. Villegas, H.E.N. Bersee, Ultrasonic welding of advanced thermoplastic composites: An investigation on energy-directing surfaces, Advances in Polymer Technology 29(2) (2010) 112-121.

[10] B. M., I.F. Villegas, H.E.N. Bersee, Optimizing ultrasonic welding of carbon-fibre reinforced polyetherimide, JEC, 2012, pp. 54-58.

[11] G. Palardy, I.F. Villegas, On the effect of flat energy directors thickness on heat generation during ultrasonic welding of thermoplastic composites, Compos. Interfaces (2016) 1-12.

[12] I.F. Villegas, G. Palardy, Ultrasonic welding of CF/PPS composites with integrated triangular energy directors: melting, flow and weld strength development, Compos. Interfaces (2016) 1-14.

[13] B. Harras, K.C. Cole, T. Vu-Khanh, Optimization of the ultrasonic welding of PEEKcarbon composites, Journal of Reinforced Plastics and Composites 15(2) (1996) 174-182.

[14] S.-J. Liu, I.T. Chang, S.-W. Hung, Factors affecting the joint strength of ultrasonically welded polypropylene composites, Polym. Compos. 22(1) (2001) 132-141.

[15] S.-J. Liu, I.-T. Chang, Optimizing the Weld Strength of Ultrasonically Welded Nylon Composites, Journal of Composite Materials 36(5) (2002) 611-624.

[16] H.-M. Lu, A. Benatar, F.-G. He, Sequential Ultrasonic Welding of PEEK/Graphite Composites Plates, ANTEC 91, 1991.

[17] G. Palardy, F.M. Agricola, C. Dransfeld, I.F. Villegas, Up-Scaling of the Ultrasonic Welding Process for Joining Thermoplastic Composites, Society for the Advancement of Material and Process Engineering, Baltimore, 2015. 
[18] F. Senders, M. van Beurden, G. Palardy, I.F. Villegas, Zero-flow: a novel approach to continuous ultrasonic welding of CF/PPS thermoplastic composite plates, Advanced Manufacturing: Polymer \& Composites Science (2016) 1-10.

[19] A. Levy, S. Le Corre, N. Chevaugeon, A. Poitou, A level set based approach for the finite element simulation of a forming process involving multiphysics coupling: Ultrasonic welding of thermoplastic composites, European Journal of Mechanics - A/Solids 30(4) (2011) 501-509.

[20] A. Levy, S. Le Corre, A. Poitou, E. Soccard, Ultrasonic welding of thermoplastic composites: modeling of the process using time homogenization, 9(1) (2011) 53-72.

[21] X. Wang, J. Yan, R. Li, S. Yang, FEM Investigation of the Temperature Field of Energy Director During Ultrasonic Welding of PEEK Composites, Journal of Thermoplastic Composite Materials 19(5) (2006) 593-607.

[22] J. Yan, X. Wang, R. Li, H. Xu, S. Yang, The Effects of Energy Director Shape on Temperature Field During Ultrasonic Welding of Thermoplastic Composites, Key Engineering Materials 353-358 (2007) 4.

[23] A. Levy, S. Le Corre, I. Fernandez Villegas, Modeling of the heating phenomena in ultrasonic welding of thermoplastic composites with flat energy directors, Journal of Materials Processing Technology 214(7) (2014) 1361-1371.

[24] A. Levy, S. Le Corre, A. Poitou, Ultrasonic welding of thermoplastic composites: a numerical analysis at the mesoscopic scale relating processing parameters, flow of polymer and quality of adhesion, International Journal of Material Forming 7(1) (2014) 39-51.

[25] C.J. Nonhof, G.A. Luiten, Estimates for process conditions during the ultrasonic welding of thermoplastics, Polymer Engineering \& Science 36(9) (1996) 1177-1183.

[26] M.N. Tolunay, P.R. Dawson, K.K. Wang, Heating and bonding mechanisms in ultrasonic welding of thermoplastics, Polymer Engineering \& Science 23(13) (1983) 726733. 


\section{Figure Captions}

Figure 1: Principle of ultrasonic welding of thermoplastic composites using a flat energy director

Figure 2: Ultrasonic welding setup used in this work. 1: Sonotrode, 2: clamp for lower adherend, 3: clamp for upper adherend, 4: moving platform, and 5: springs supporting the moving platform.

Figure 3: Determination of amplitude transmission via displacement measurement of the upper adherend during ultrasonic welding using a tilted sensor. Measured displacement: $D_{\text {meas }}$, real displacement: $D$, sensor tilt angle: $\alpha$, and distance between sonotrode and laser: $d$. Dimensions are not to scale.

Figure 4: Boundary conditions of 2D model to predict the power dissipated during the ultrasonic welding process. A quarter model was used based on the symmetry (Sym.) of the system. Dimensions are not to scale.

Figure 5: Displacement sensor positions on the bottom surface of the sonotrode for calibration measurements.

Figure 6: (a) Representative example of the measured sonotrode displacement using a laser sensor at position 1 (as shown in Figure 5) with a nominal amplitude of $45.4 \mu \mathrm{m}$. (b) Corresponding Fast Fourier Transformation (FFT).

Figure 7: Distances $d$ from the sonotrode at which displacement was measured by the laser sensor, as in Figure 3.

Figure 8: (a) Displacement curve measured by the sensor on the upper adherend at $d$ $=2 \mathrm{~mm}$, the dashed, red line represents the displacement of the sonotrode provided by the USW machine. Similar curves are shown for other $d$ values: (b) $5 \mathrm{~mm}$, (c) $15 \mathrm{~mm}$ and (d) $25 \mathrm{~mm}$. In all cases, the welding force and nominal amplitude were $1500 \mathrm{~N}$ and $45.4 \mu \mathrm{m}$, respectively.

Figure 9: Average amplitude of the displacement curves over the entire duration of the vibration phase for all $d$ values shown in Figure 8. Experimental data points are compared with numerical dynamic simulations for a nominal amplitude of the sonotrode of $45.4 \mu \mathrm{m}$.

Figure 10: Fast Fourier Transform (FFT) of representative example of sensor measurements for displacement of the upper adherend at a distance $\mathrm{d}=2 \mathrm{~mm}$ from the sonotrode (as shown in Figure 3).

Figure 11: (a) Representative displacement curve measured using the laser sensor at $\mathrm{d}=2 \mathrm{~mm}$ on the upper adherend during welding (1500 $\mathrm{N}$ welding force and $36.3 \mu \mathrm{m}$ nominal amplitude), and (b) Close-up of the rectangular area in (a) 
where the black dashed line represents the estimated mean displacement curve.

Figure 12: Evolution of the estimated amplitude transmitted to the upper adherend ( $1500 \mathrm{~N}$ welding force and $36.3 \mu \mathrm{m}$ nominal amplitude). Values were calculated at $0.004 \mathrm{~s}$ time intervals over the duration of the process, an affine function was fitted through the data points and a linear increase of the amplitude was proposed during the period needed for full amplitude to be established in the sonotrode.

Figure 13: Comparison between experimental (Exp power) and simulated (Total heating) power curves for a representative welding case $(1500 \mathrm{~N}$ welding force and $36.3 \mu \mathrm{m}$ nominal amplitude) using average amplitude from Figure 12. . Viscoelastic heating from adherends: Ad Viscoelastic heating, viscoelastic heating from energy director: ED Viscoelastic heating, frictional heating: Friction heating. The circled areas represent two characteristic power peaks for both simulated and experimental curves.

Figure 14: Predicted temperature profile across the thickness of energy director and upper adherend over a vertical distance of $1 \mathrm{~mm}$ at three locations: overlap centreline (--), overlap quarter (-) and overlap edge (-). (a) $t=0.12 \mathrm{~s}$ (left circled peak in Figure 13), and (b) $t=0.50 \mathrm{~s}$ (right circled peak in Figure 13).

Figure A1: Displacement of the sonotrode and upper adherend versus time. The sonotrode displacement, $u_{\text {sono }}$, is a sine (with a period $\mathrm{T}$ and an amplitude ). However, due to the hammering effect, the displacement of the upper adherend, $u_{i m p}$, is truncated with a loss of contact time tc.

Figure A2: Viscoelastic efficiency e vs amplitude transfer ratio $\boldsymbol{\alpha}_{\boldsymbol{h}}$. The viscoelastic efficiency quantifies how much the viscoelastic heating, crucial to the welding process, is reduced because of the hammering effect. The amplitude transfer ratio $\boldsymbol{\alpha}_{\boldsymbol{h}}$ is the ratio between the amplitude $\boldsymbol{a}_{\boldsymbol{a d h}}$ of the vibration transmitted to the upper substrate and the sonotrode amplitude $\boldsymbol{a}_{\text {sono }}$. The contact time ratio $\boldsymbol{\alpha}$ is the ratio of contact duration over the period. The process efficiency (in terms of energy dissipation) appears as roughly proportional to the amplitude transmitted. 


\title{
A study on amplitude transmission in ultrasonic welding of thermoplastic composites,
}

\author{
Genevieve Palardy ${ }^{3}$, Huajie Shi ${ }^{1}$, Arthur Levy ${ }^{2}$, Steven LeCorre ${ }^{2}$ \\ and Irene Fernandez Villegas ${ }^{1}$
}

\section{Figures}




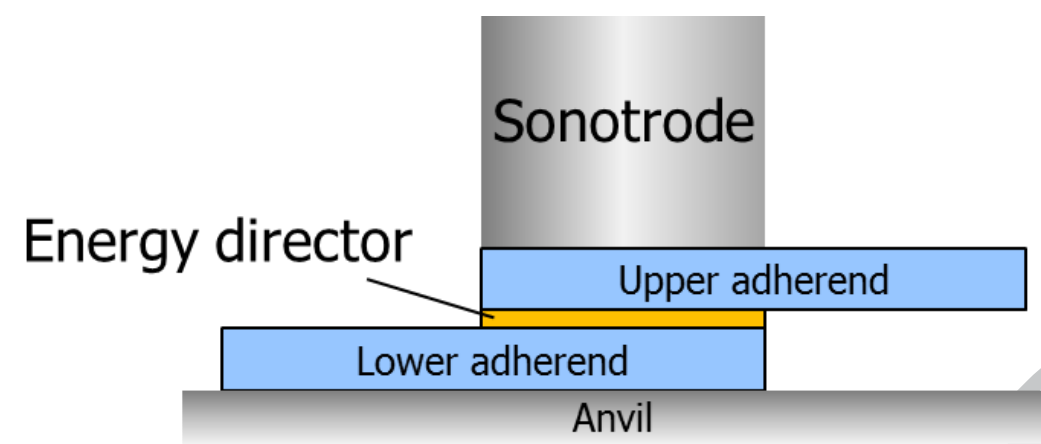

Figure 7: Principle of ultrasonic welding of thermoplastic composites using a flat energy director.

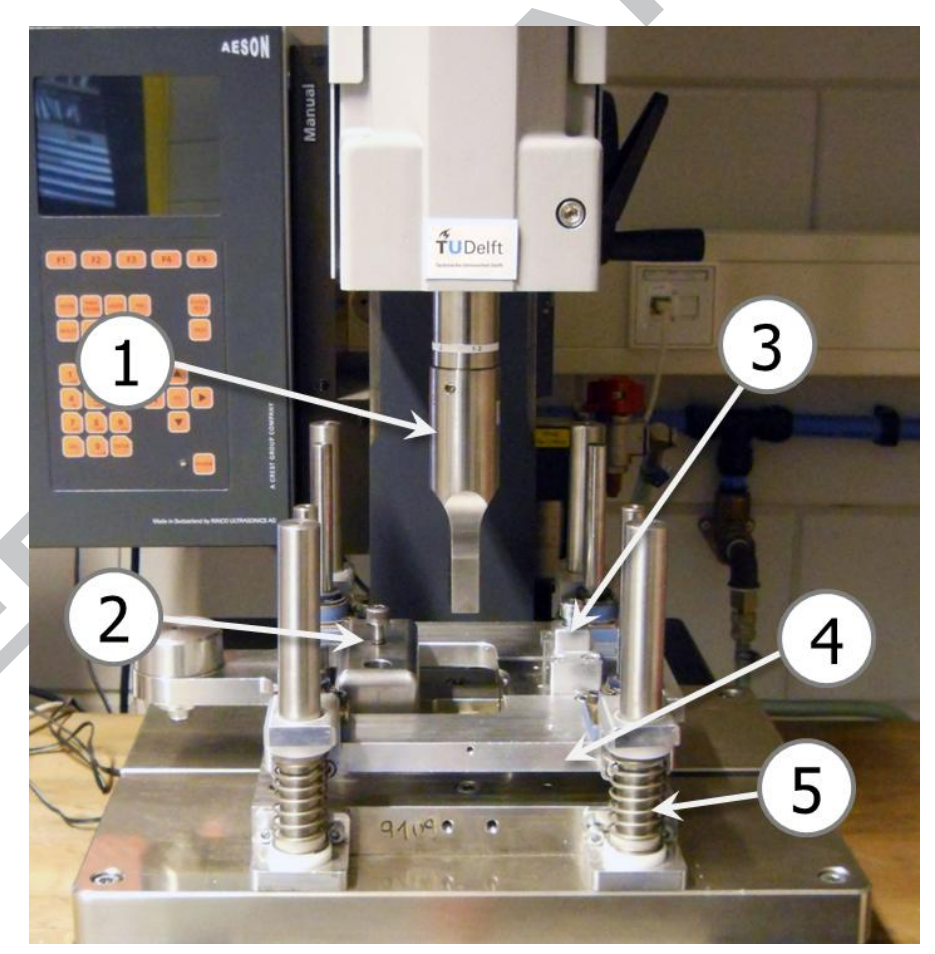

Figure 8: Ultrasonic welding setup used in this work. 1: Sonotrode, 2: clamp for lower adherend, 3: clamp for upper adherend, 4: moving platform, and 5: springs supporting the moving platform. 


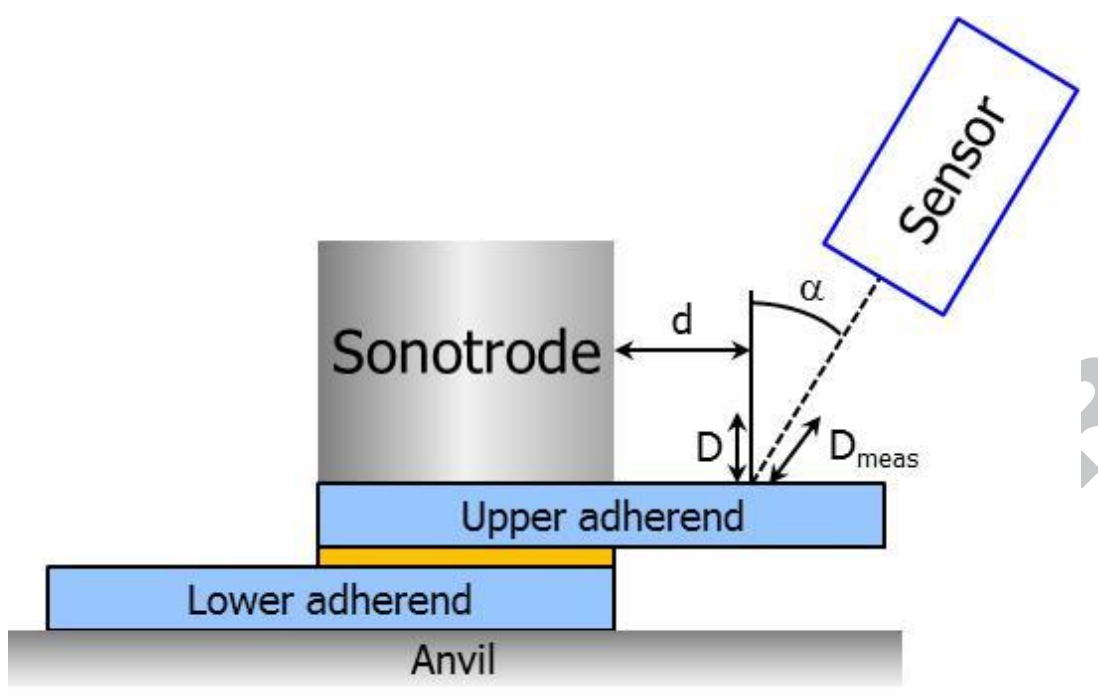

Figure 9: Determination of amplitude transmission via displacement measurement of the upper adherend during ultrasonic welding using a tilted sensor. Measured displacement: $D_{\text {meas }}$, real displacement: $D$, sensor tilt angle: $\alpha$, and distance between sonotrode and laser: $d$. Dimensions are not to scale.

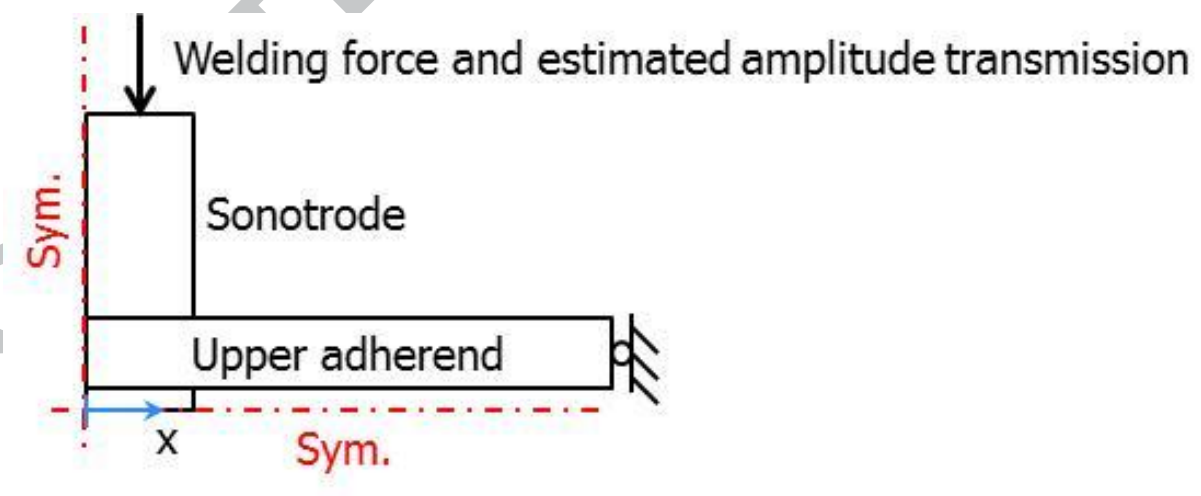

Figure 10: Boundary conditions of 2D model to predict the power dissipated during the ultrasonic welding process. A quarter model was used based on the symmetry (Sym.) of the system. Dimensions are not to scale. 


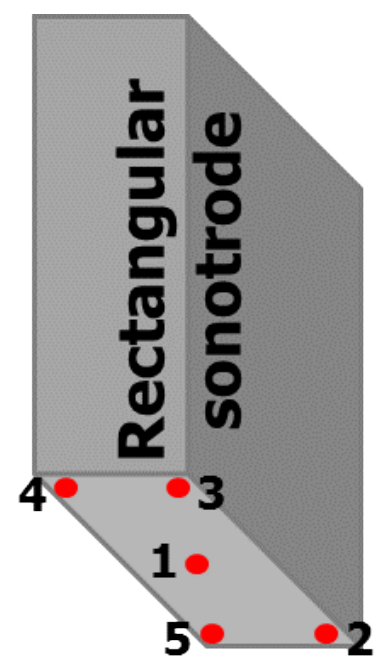

Figure 11: Displacement sensor positions on the bottom surface of the sonotrode for calibration measurements. 


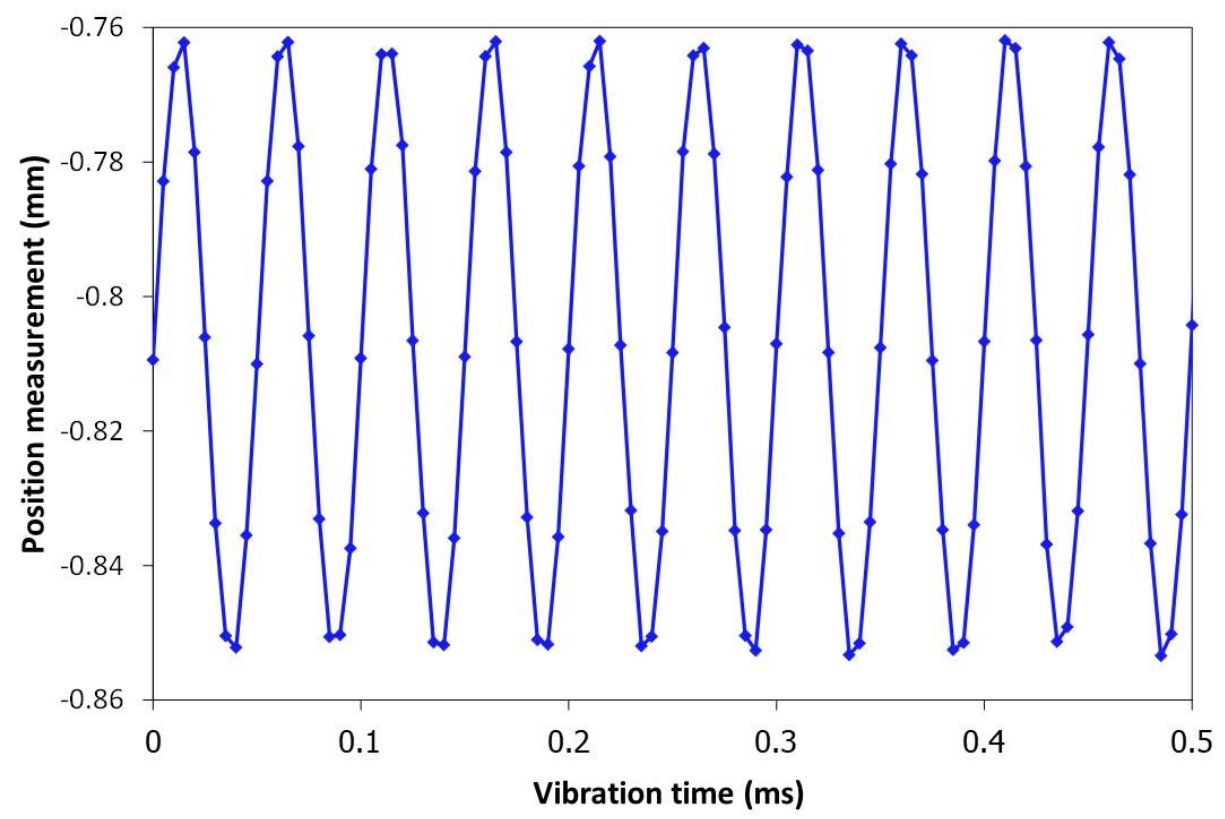

(a)

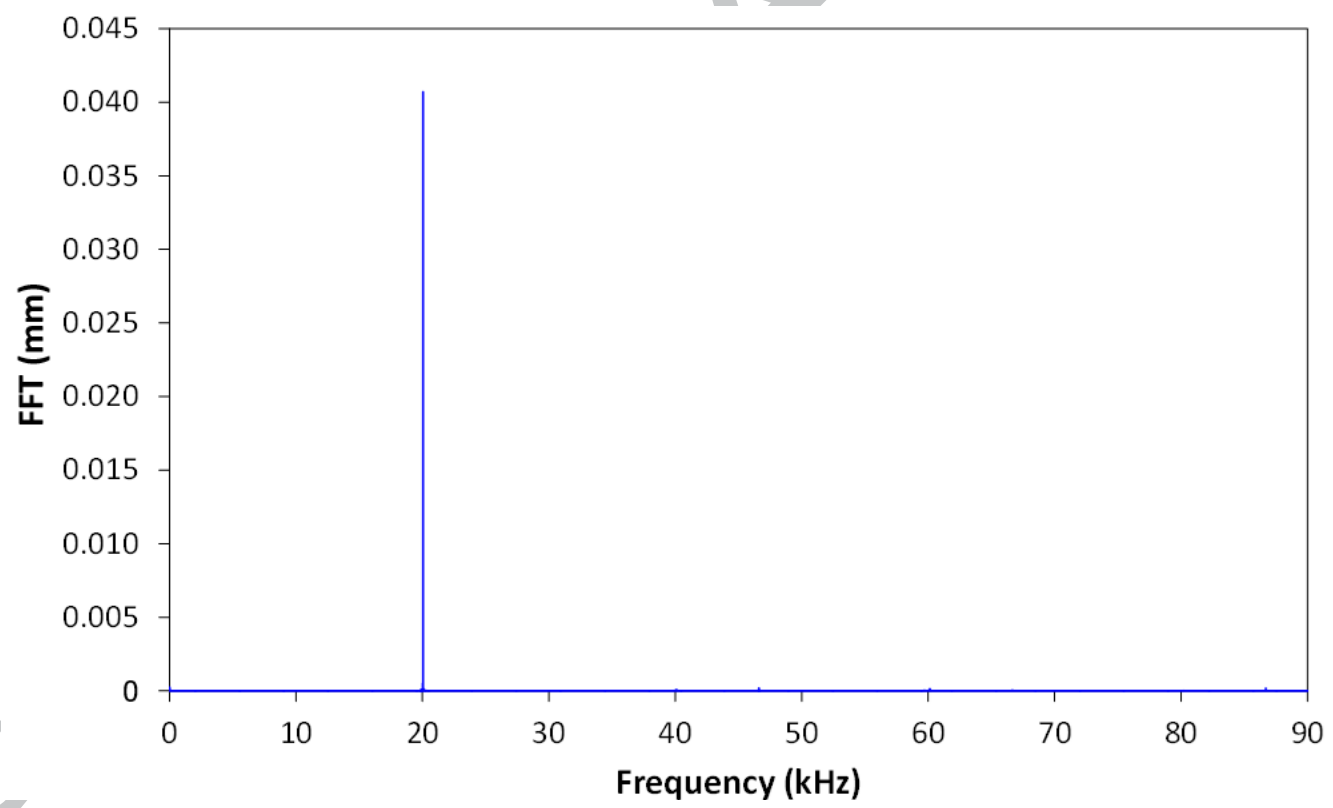

(b)

Figure 12: (a) Representative example of the measured sonotrode displacement using a laser sensor at position 1 (as shown in Figure 11) with a nominal amplitude of $45.4 \mu \mathrm{m}$. (b) Corresponding Fast Fourier Transformation (FFT). 


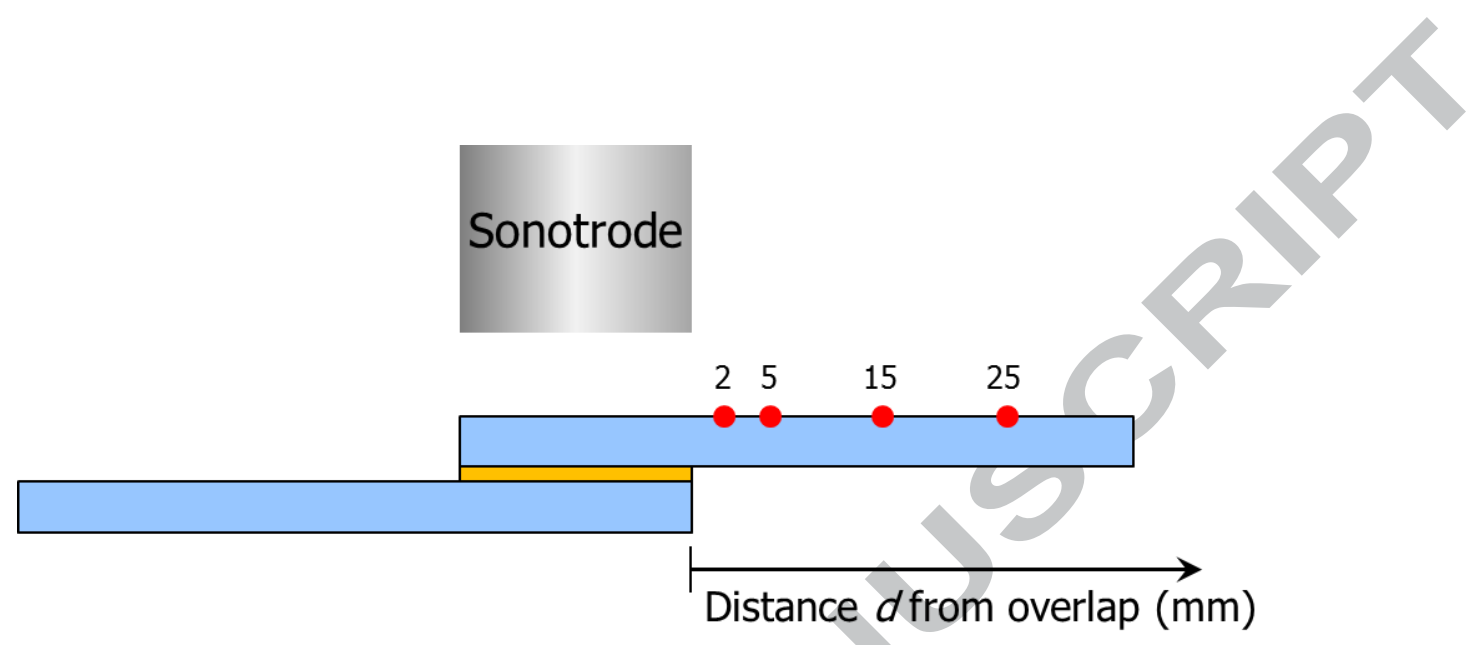

Figure 7: Distances $d$ from the sonotrode at which displacement was measured by the laser sensor, as shown in Figure 3. 

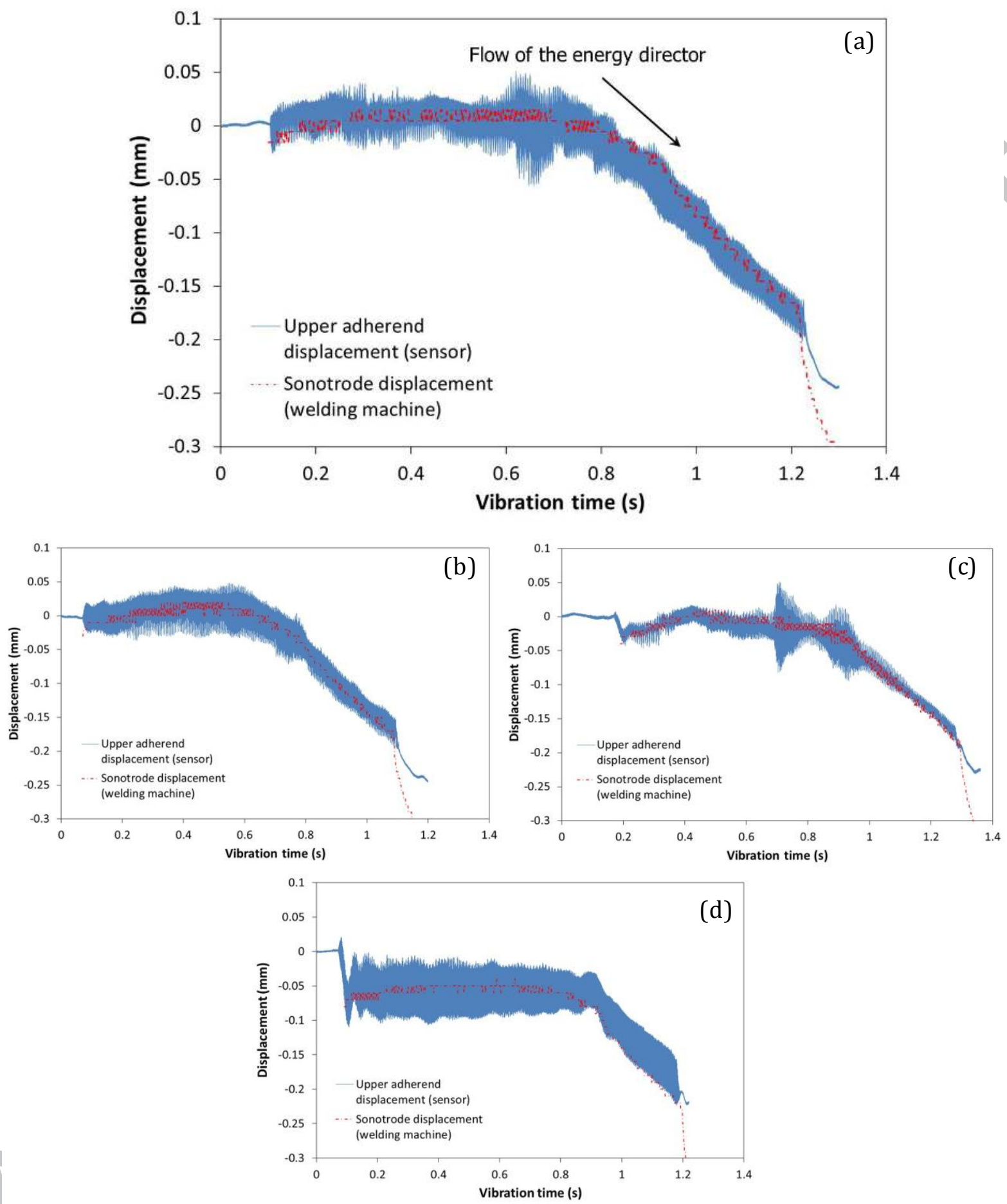

Figure 8: (a) Displacement curve measured by the sensor on the upper adherend at $d=2 \mathrm{~mm}$, the dashed, red line represents the displacement of the sonotrode provided by the USW machine. Similar curves are shown for other $d$ values: (b) $5 \mathrm{~mm}$, (c) $15 \mathrm{~mm}$ and (d) $25 \mathrm{~mm}$. In all cases, the welding force and nominal amplitude were $1500 \mathrm{~N}$ and $45.4 \mu \mathrm{m}$, respectively. 


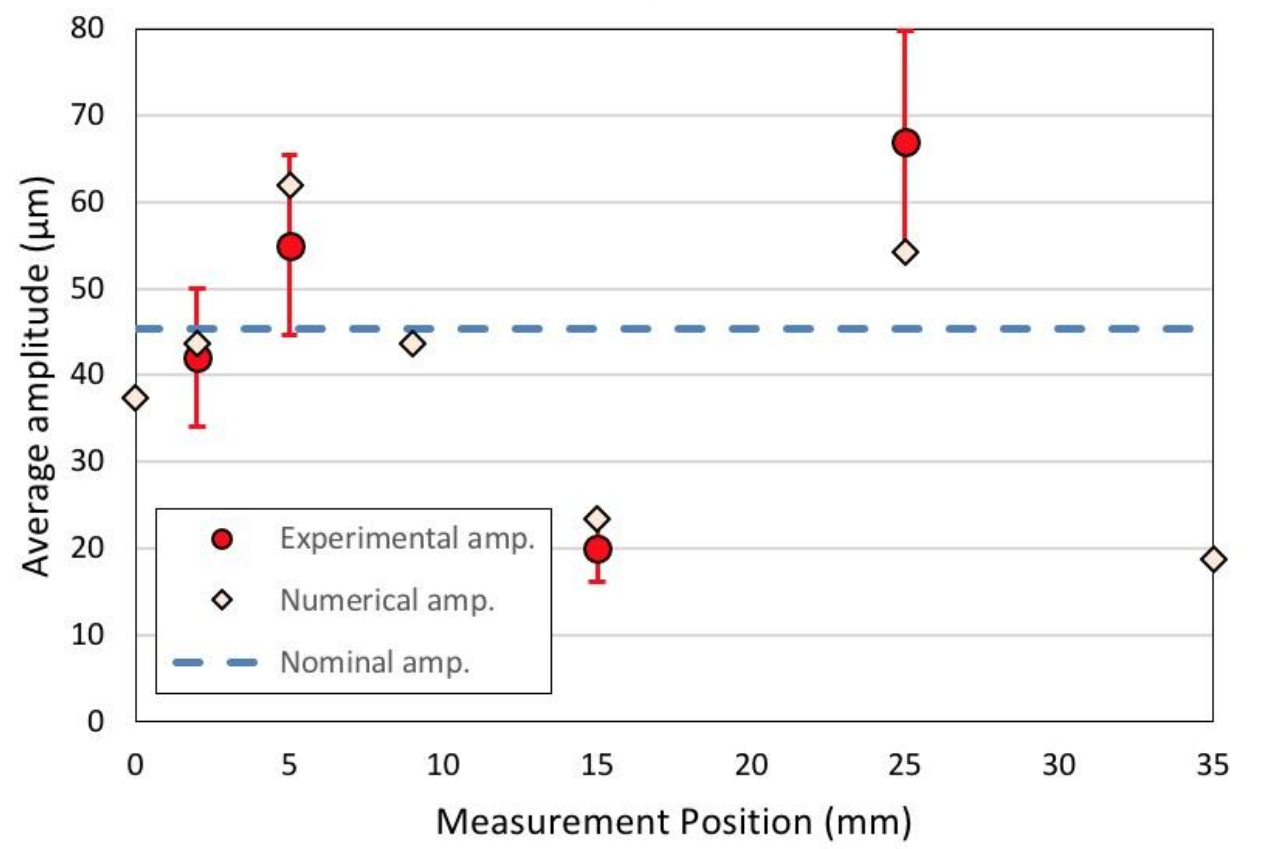

Figure 9: Average amplitude of the displacement curves over the entire duration of the vibration phase for all $d$ values shown in Figure 8. Experimental data points are compared with numerical dynamic simulations for a nominal sonotrode amplitude of $45.4 \mu \mathrm{m}$. 


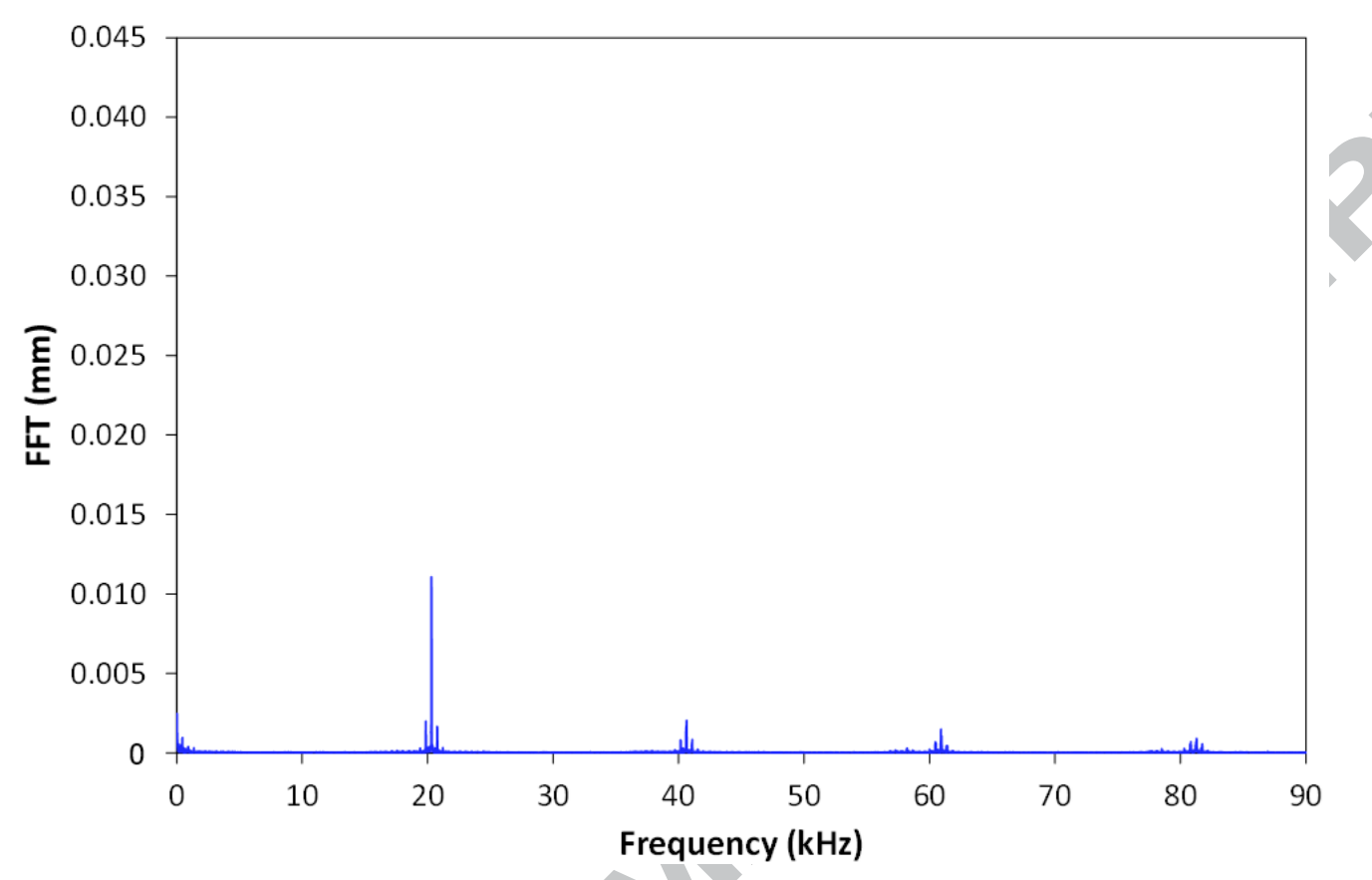

Figure 10: Fast Fourier Transform (FFT) of representative example of sensor measurements for displacement of the upper adherend at a distance $d=2 \mathrm{~mm}$ from the sonotrode (as shown in Figure 3 ). 


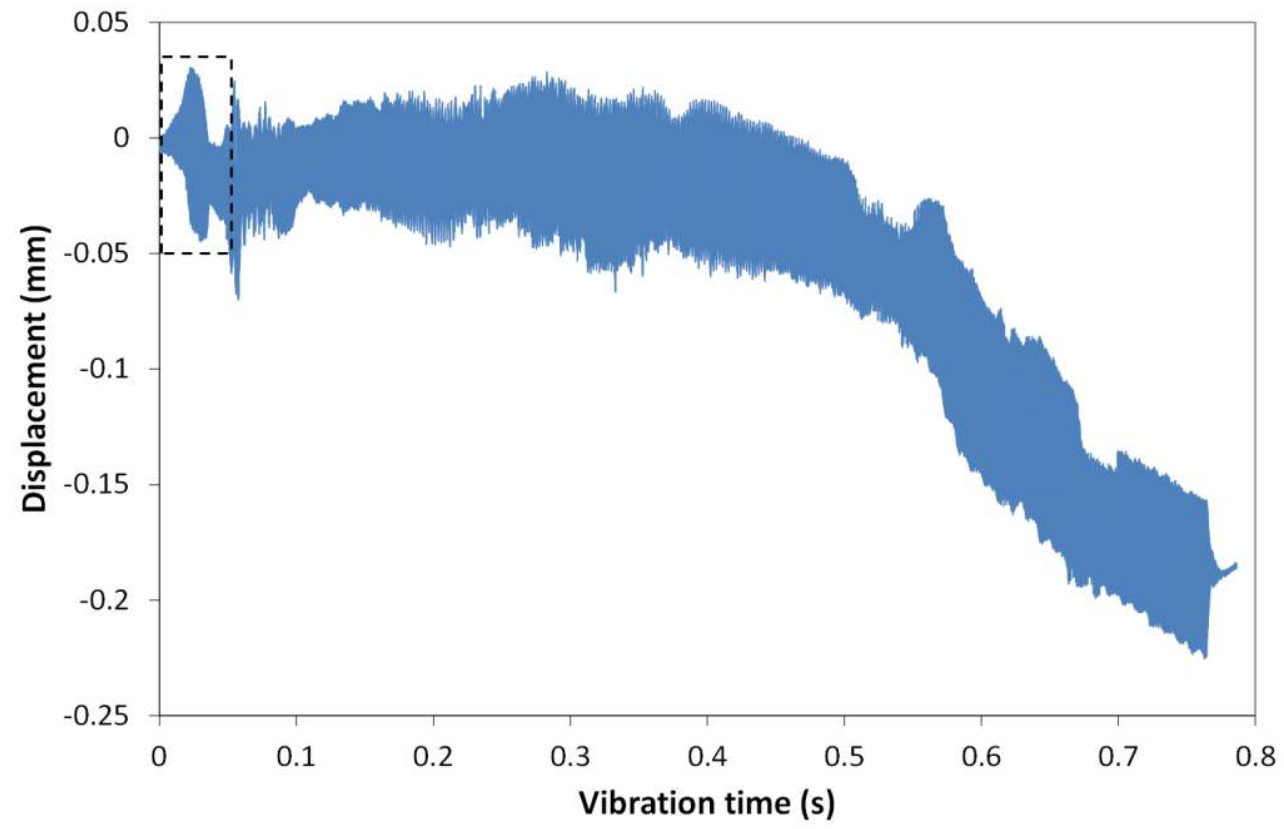

(a)

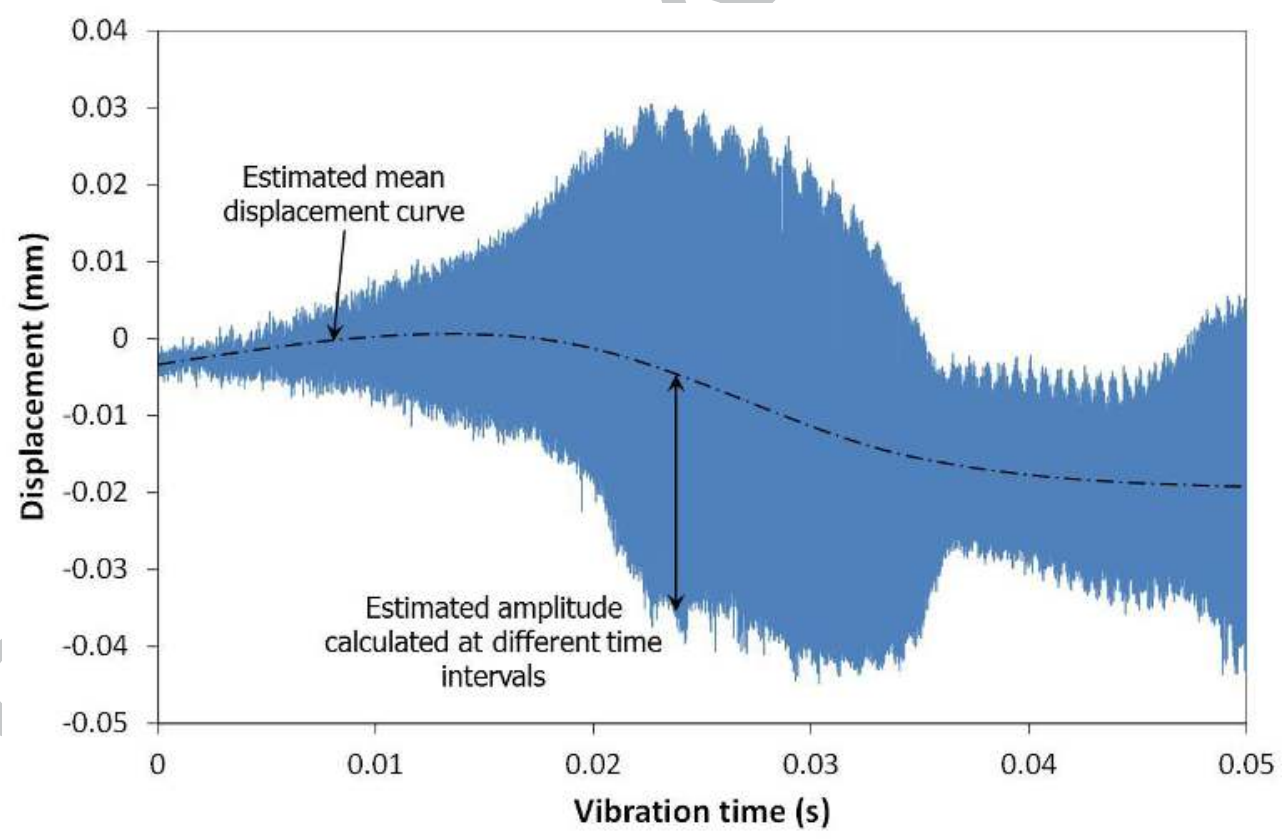

(b)

Figure 11: (a) Representative displacement curve measured using the laser sensor at $d=2 \mathrm{~mm}$ on the upper adherend during welding ( $1500 \mathrm{~N}$ welding force and $36.3 \mu \mathrm{m}$ nominal amplitude), and (b) Close-up of the rectangular area in (a) where the black dashed line represents the estimated mean displacement curve. 


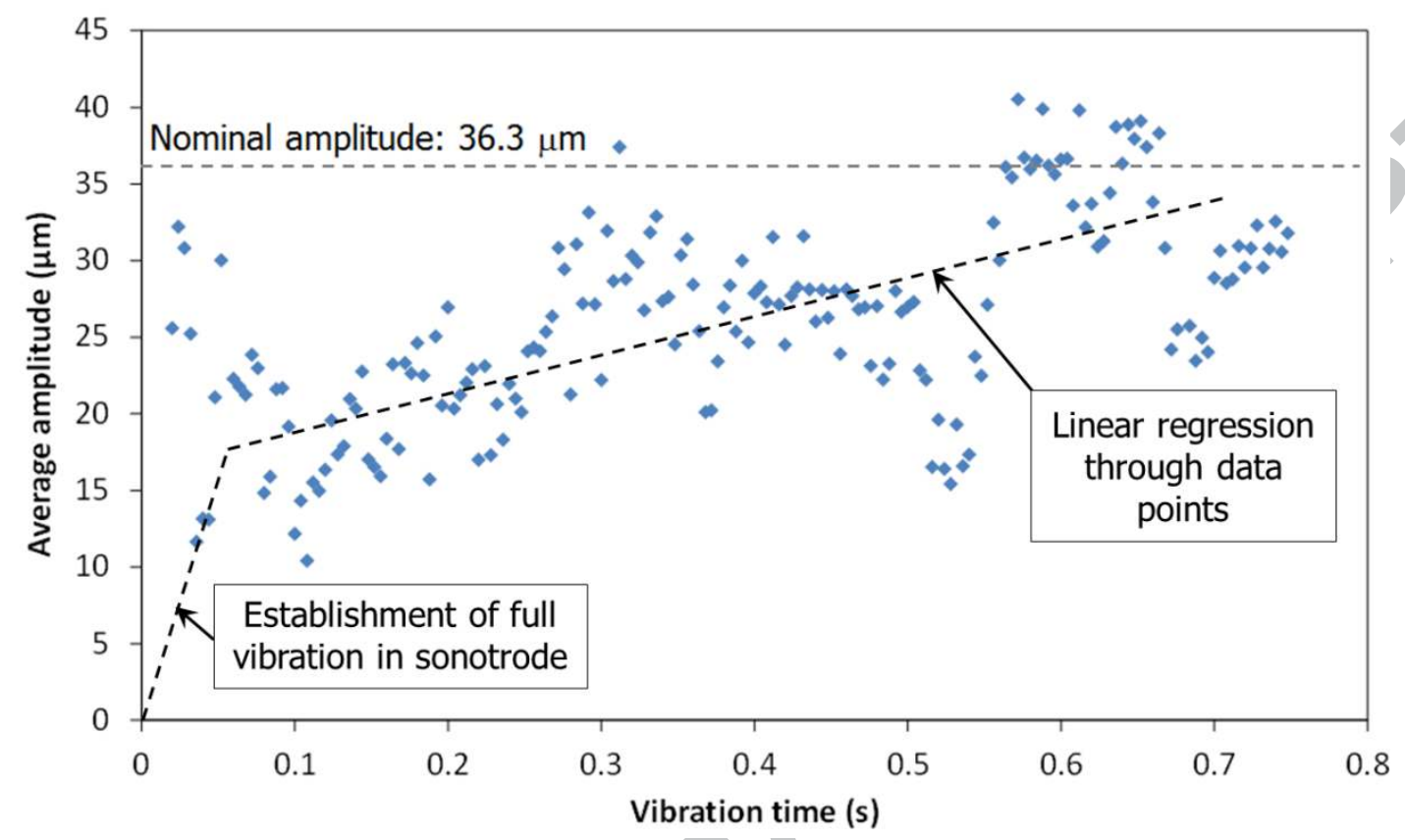

Figure 12: Evolution of the estimated amplitude transmitted to the upper adherend (1500 N welding force and $36.3 \mu \mathrm{m}$ nominal amplitude). Values were calculated at $0.004 \mathrm{~s}$ time intervals over the duration of the process, an affine function was fitted through the data points and a linear increase of the amplitude was proposed during the period needed for full amplitude to be established in the sonotrode. 


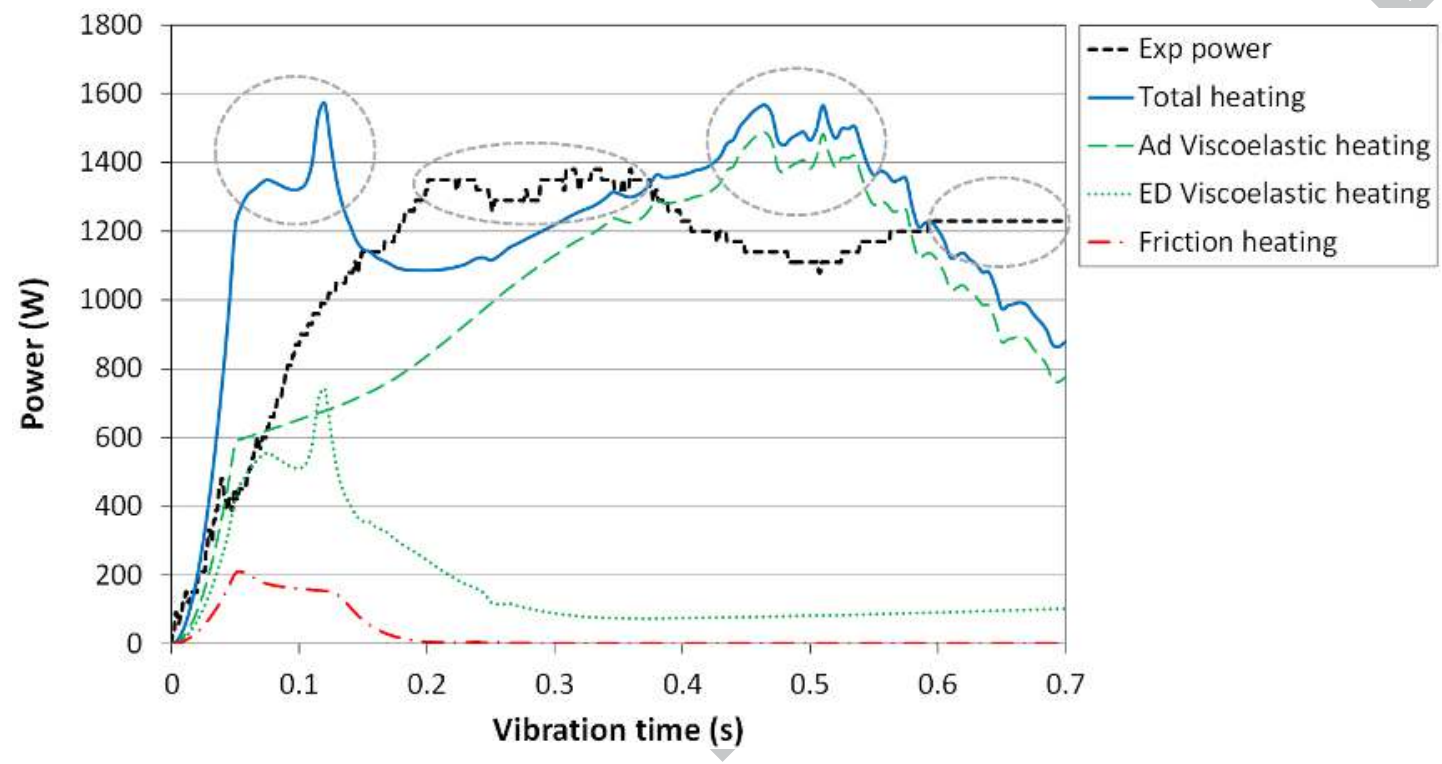

Figure 13: Comparison between experimental (Exp power) and simulated (Total heating) power curves for a representative welding case ( $1500 \mathrm{~N}$ welding force and $36.3 \mu \mathrm{m}$ nominal amplitude) using average amplitude from Figure 12. Viscoelastic heating from adherends: Ad Viscoelastic heating, viscoelastic heating from energy director: ED Viscoelastic heating, frictional heating: Friction heating. The circled areas represent two characteristic power peaks for both simulated and experimental curves. 


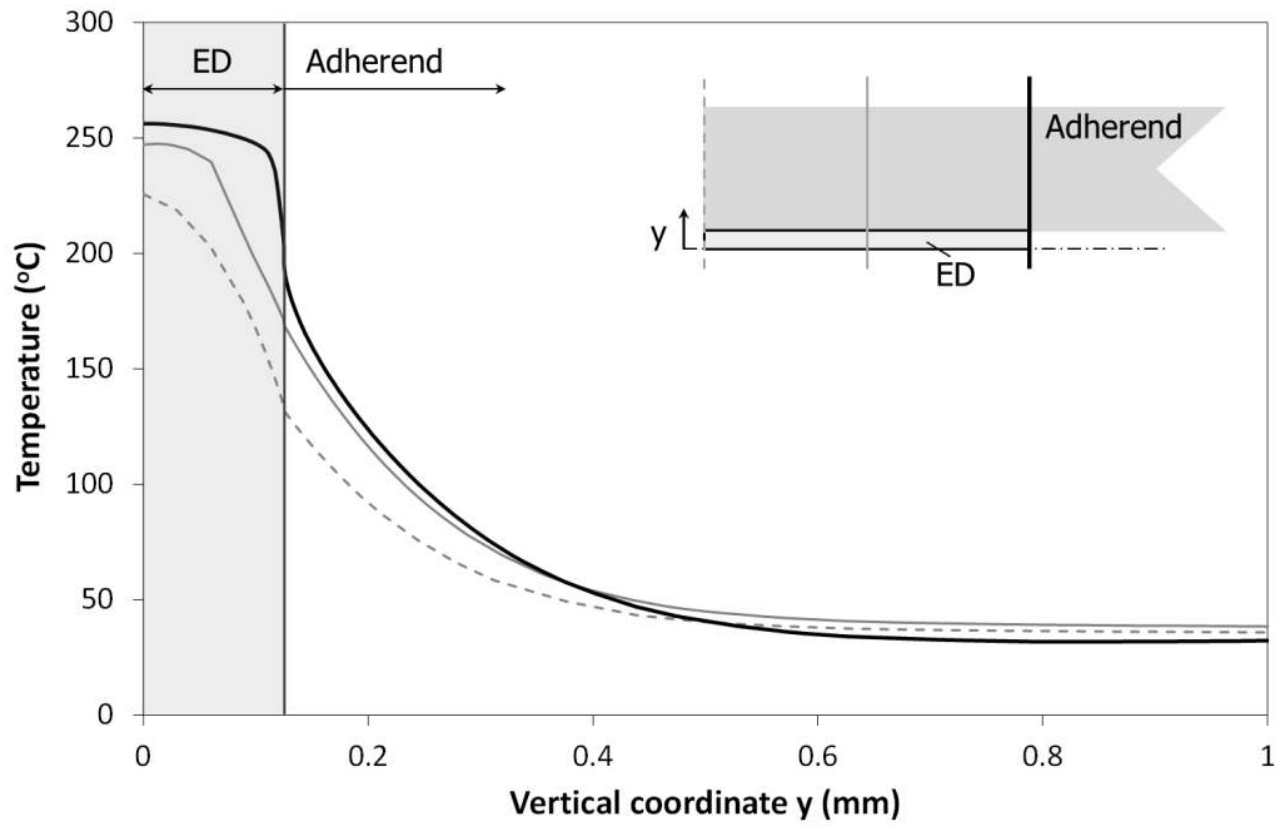

(a)

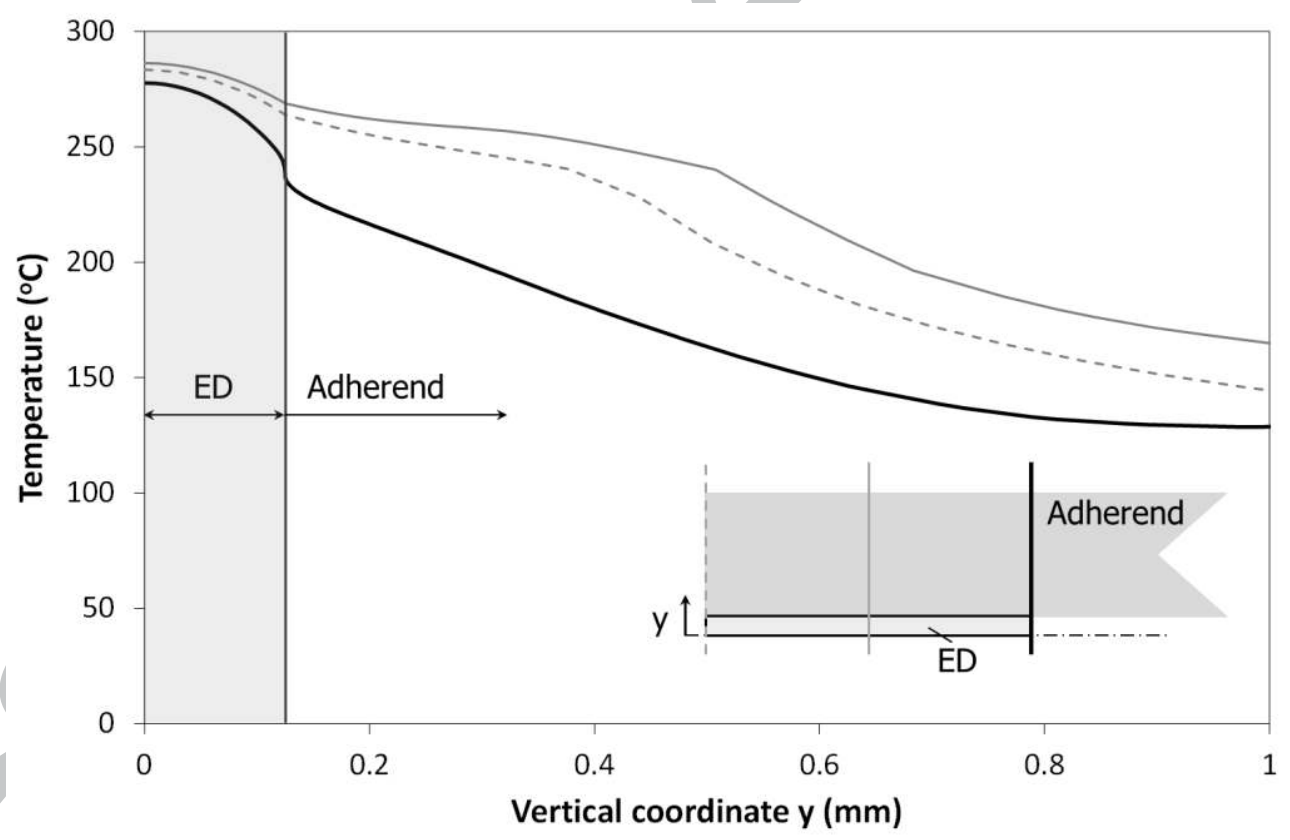

(b)

Figure 14: Predicted temperature profile across the thickness of energy director and upper adherend over a vertical distance of $1 \mathrm{~mm}$ at three locations: overlap centreline (--), overlap quarter $(-)$ and overlap edge (-). (a) $t=0.12 \mathrm{~s}$ (left circled peak in Figure 13), and (b) $t=0.50 \mathrm{~s}$ (right circled peak in Figure 13). 


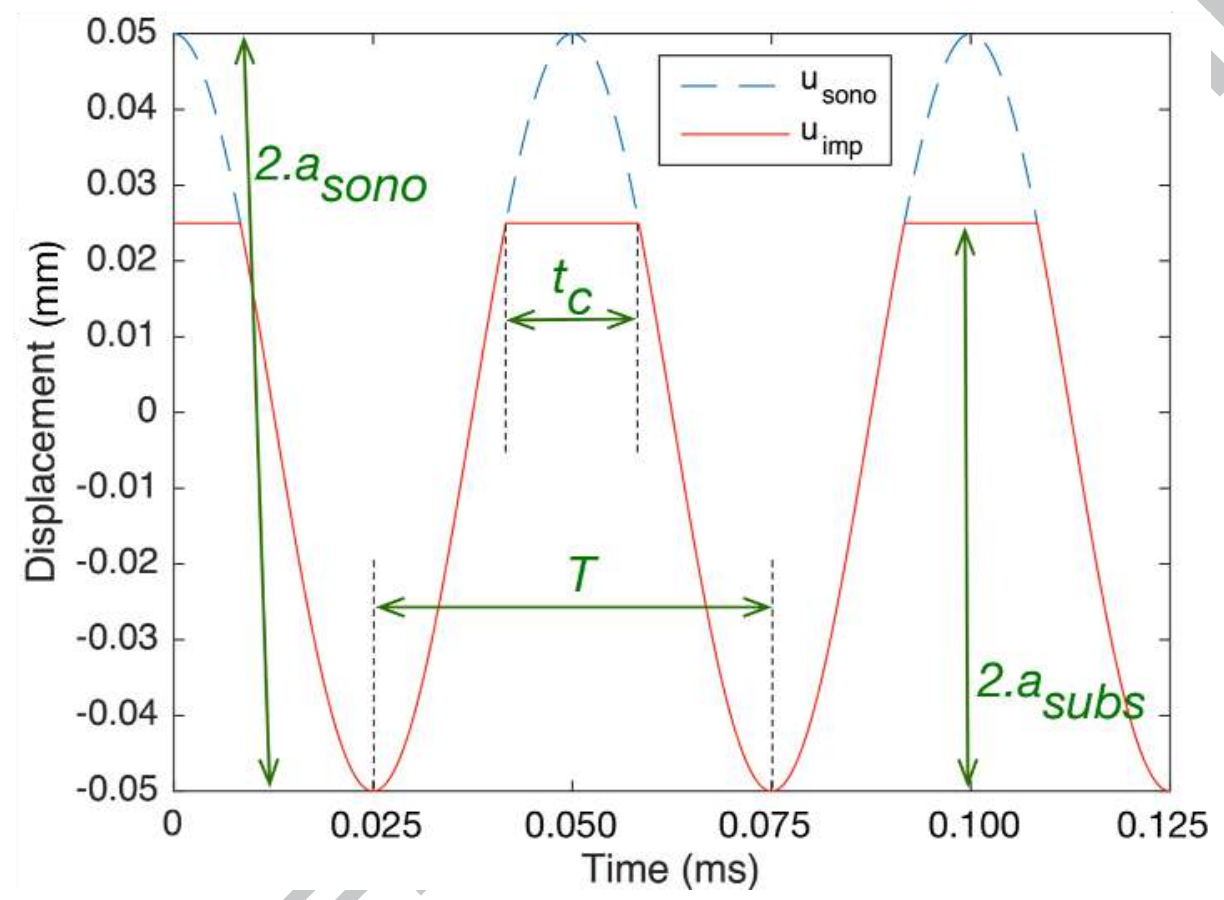

Figure A1: Displacement of the sonotrode and upper adherend versus time. The sonotrode displacement, $u_{\text {sono }}$, is a sine (with a period T and an amplitude $a_{\text {sono }}$ ). However, due to the hammering effect, the displacement of the upper adherend, $u_{i m p}$, is truncated with a loss of contact time $t_{c}$. 


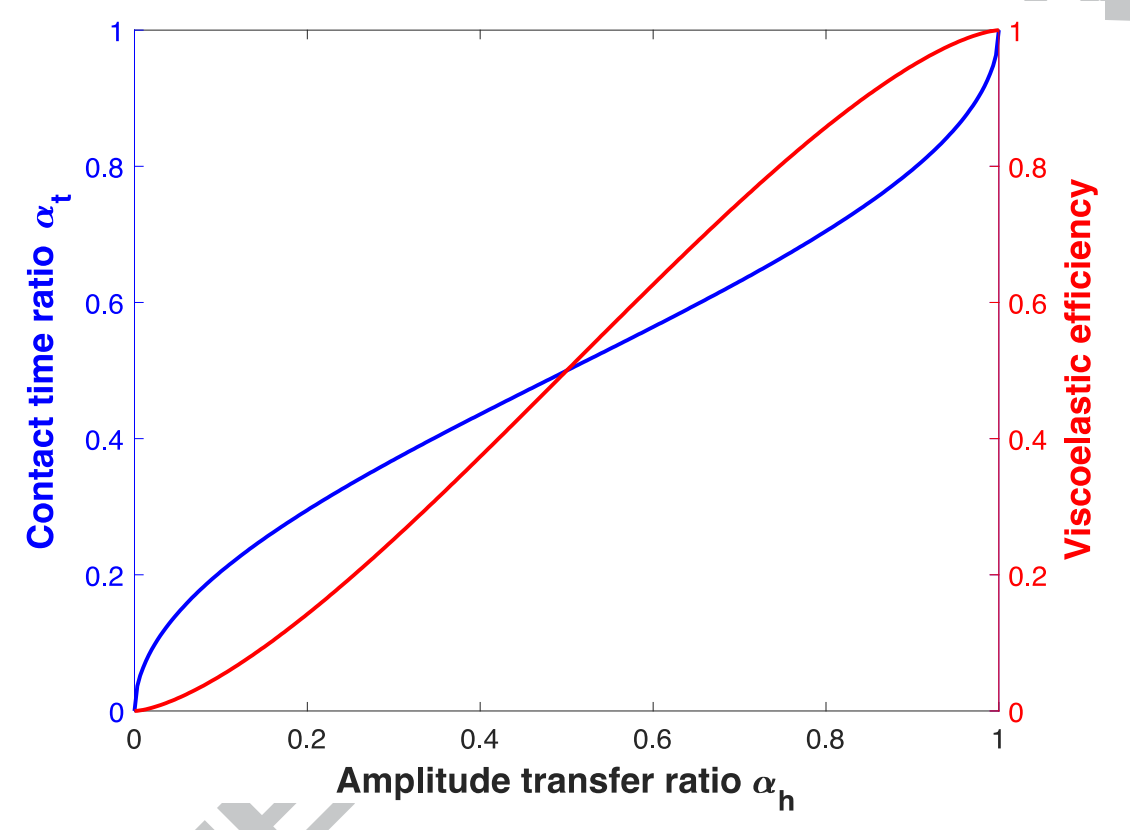

Figure A2: Viscoelastic efficiency e vs amplitude transfer ratio $\boldsymbol{\alpha}_{\boldsymbol{h}}$. The viscoelastic efficiency quantifies how much the viscoelastic heating, crucial to the welding process, is reduced because of the hammering effect. The amplitude transfer ratio is the ratio between the amplitude of the vibration transmitted to the upper substrate and the sonotrode amplitude . The contact time ratio is the ratio of contact duration over the period. The process efficiency (in terms of energy dissipation) appears as roughly proportional to the amplitude transmitted. 